\title{
Application of Organic Amendments to Cadmium-contaminated Soils for Phytostabilization Potential by Dicotyledonous Bioenergy Crops
}

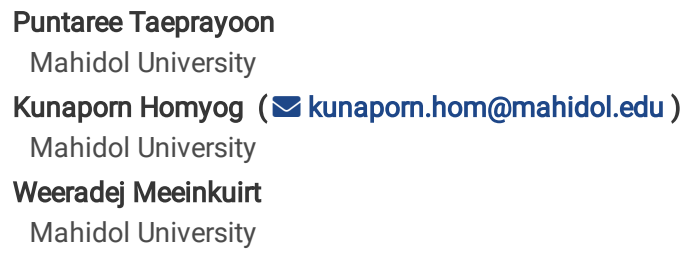

\section{Research Article}

Keywords: Acacia (Acacia mangium), jatropha (Jatropha curcas), cassava (Manihot esculenta), phytomanagement

Posted Date: December 22nd, 2021

DOI: https://doi.org/10.21203/rs.3.rs-1160309/v1

License: (c) (i) This work is licensed under a Creative Commons Attribution 4.0 International License. Read Full License 


\section{Abstract}

Acacia (Acacia mangium), jatropha (Jatropha curcas), and cassava (Manihot esculenta) were cultivated in a greenhouse to see how organic amendments affected plant survival and accumulation of cadmium (Cd) in plant tissues. The study plants are bioenergy crops, which are advantageous to phytomanagement because they provide a significant economic benefit to local residents and farmers in the agricultural sector while also simultaneously reducing Cd entry into food webs through consumption. In this study, bone meal/bat manure and leonardite/bat manure were the key organic amendments that promoted the best growth performances in acacia (growth rate in dry biomass; GRDB 24.2) and cassava (GRDB 22.2), respectively, while jatropha preferred bone meal (GRDB 17.2). However, leonardite/bone meal considerably reduced Cd uptake values in whole plant tissues of acacia and cassava (35 mg plant $^{-1}$ and 119.1 mg plant $^{-1}$, respectively), while bone meal/chicken manure greatly reduced Cd uptake values in jatropha (127.8 mg plant $\left.{ }^{-1}\right)$. Cassava is a Cd excluder; however, it may not be a useful bioenergy crop to cultivate in Cd soil because it displayed toxicity symptoms after harvesting. The best plant for phytomanagement in this study was jatropha, which demonstrated substantial growth biomass and Cd accumulation, followed by acacia.

\section{Introduction}

In both developed and developing countries, soil contamination with high concentrations of heavy metals, particularly toxic metals such as cadmium (Cd) and lead $(\mathrm{Pb})$, in the environment, primarily caused by anthropogenic activities such as households and industries, e.g., mining and metal production sectors, has become a serious problem. Heavy metal accumulation in agricultural soils has a deleterious impact on crop growth and development, resulting in decreased crop yields ${ }^{1}$. Furthermore, bioaccumulation and tropic transfer of heavy metals in the food chains end up posing a major health concern to living organisms. When all toxic metals' effects on public health are considered, $\mathrm{Cd}$ is the most important toxic metal because it has sublethal, behavioral, carcinogenic, and mutagenic effects on humans, even at low concentrations ${ }^{2}$.

Chemical precipitation, filtration, ion exchange, membrane filtration, solvent extraction, and electrochemical removal are just a few of the traditional technologies that have been employed for decades and have shown great promise in removing heavy metals from polluted areas. However, when these methods are applied, they frequently result in the production of toxic sludge and heavy metals, as well as high-energy demands and insufficient removal. These techniques are expensive, harmful to soil structure and function, and time-consuming ${ }^{3}$. Phytoremediation is a recently developed technology and a viable alternative way for controlling numerous toxic pollutants in soils, surface streams, and groundwaters using green plants ${ }^{4}$. Plant species for phytoremediation can potentially be improved by introducing soil amendments to overcome some limitations of phytoremediation constraints, hence improving the remediation capability of the most suitable plants. Organic amendments are deemed acceptable in many large-scale polluted environments because they help to improve plant growth and excluder potential while being low cost and simple to apply. Pig and cattle manures, leonardite, and other organic additions are helpful in Cd-contaminated soils ${ }^{5,6}$.

Based on the potential of plant root systems and the application of organic amendments that effectively immobilize heavy metals in soils via absorption and accumulation mechanisms by plant roots, phytostabilization is considered the most widely used phytoremediation technique initiated in the last decade ${ }^{7}$. Many studies have found that dicotyledonous plants are better suited for phytoremediation than monocotyledonous plants because they have higher biomass, longer life, and hence a greater ability to accumulate heavy metals ${ }^{8}$. Many dicotyledonous crop plants, such as acacia (Acacia mangium Willd.) for nickel (Ni) and chromium ( $\mathrm{Cr}$ ) and jatropha (Jatropha curcas L.) for copper (Cu), zinc ( $\mathrm{Zn}), \mathrm{Cd}$, and lead (Pb), exhibited much greater heavy metal accumulation in roots compared with those obtained from shoots. These excluders can be employed using the phytostabilization approach because they limit heavy metal uptake in their shoots ${ }^{9}$, whereas cassava (Manihot esculenta Crantz) is a hyperaccumulator and is employed in mercury ( $\mathrm{Hg}$ ) and gold (Au) phytoextraction because it accumulates a significant amount of $\mathrm{Hg}$ and $\mathrm{Au}$ in its shoots ${ }^{10}$.

In this study, the bioenergy crops [acacia (A. mangium), jatropha (J. curcas), and cassava (M. esculenta)] were planted in Cd-contaminated soils under greenhouse conditions to determine their phytoremediation capability and the influence of organic amendments.

\section{Materials And Methods}

Plant samples Seedlings of acacia and cassava stem cuttings were purchased from local farms in Nakhon Sawan Province, whereas seedlings of jatropha were obtained from a local farm in Phitsanulok Province, Thailand. All plant materials were cultivated in a greenhouse under controlled conditions $\left(27\right.$ to $29^{\circ} \mathrm{C}$; 63 to $75 \%$ relative humidity; 10,253 to 15,631 lux light intensity; and a 12/12-h photoperiod) before testing in pot systems.

Pot experimental design All investigations were conducted according to local guidelines ensuring ethically conducted research. Soil samples for this study were purchased from an agricultural shop in Nakhon Sawan Province, Thailand. To make a composite soil, they were properly mixed and then ground with an agate mortar and pestle before passing through a 2-mm sieve. Two and one-half $\mathrm{kg}$ of soil sample was mixed with an amendment and left to settle for at least one month in a plastic pot with a diameter of 10 inches and a height of 7.5 inches. In the soil investigated, the ratio of each amendment in the tested soils was $3: 17$, or $15 \%$ of the total soil weight. Commercial soil (C0) purchased from a local agricultural shop in Nakhon Sawan Province was used for mixing with an organic amendment. Organic soil amendments and their weight were applied to soils in the following order: $375 \mathrm{~g}$ leonardite (termed SL), $375 \mathrm{~g}$ bone meal (SBM), $187.5 \mathrm{~g}$ leonardite/187.5 g bone meal (SLBM), $250 \mathrm{~g}$ leonardite/125 g vermicompost (SLVC), $250 \mathrm{~g}$ leonardite/125 g chicken manure (LCM), 250 $\mathrm{g}$ leonardite/125 g bat manure (LBM), $250 \mathrm{~g}$ bone meal/125 g vermicompost (BMVC), $250 \mathrm{~g}$ bone meal/125 g chicken manure (BMCM) and $250 \mathrm{~g}$ bone meal/125 g bat manure (BMBM). After soil mixing, soil texture, organic matter (OM), cation exchange capacity (CEC), total nitrogen (total $\mathrm{N}$ ), extractable phosphorus (Ext. P), extractable potassium (Ext. K), extractable calcium (Ext. Ca) and extractable magnesium (Ext. Mg) were measured using the procedures of APHA, AWWA, and WEF ${ }^{11}$. A pH meter (Accumet AP115 pH meter) and an EC meter (Hanna instruments; HI 993310) were used to measure the pH and 
electrical conductivity $(E C)$ of the soil in a $1: 5$ soil/water solution. The procedure for water saturation in soils from 70 to $80 \%$ was carried out using the method of Blaylock et al. ${ }^{12}$.

One replication was represented by a single pot with a single plant. Each treatment had four replicates. Pots were set in a randomized complete block design (RCBD) on fixed benches. To maintain proper nutrients and moisture in soils, plants were watered daily with deionized (DI) water and supplemented with $6 \mathrm{~g}$ Osmocote $\rightarrow$ for each pot at 1.5 months.

Cadmium determination in plant and soil Plants were carefully removed from the pots after harvesting bioenergy crops at three months. They were washed thoroughly with tap water to remove excess soil, and rinsed with distilled water and DI water, respectively. Plants were mostly divided in leaves, stems and roots and then packed in paper bags, labeled, and oven-dried for five days at $70^{\circ} \mathrm{C}$. To make a fine powder, plant tissues were weighed, ground with a mortar and pestle (IKA; A11 basic) and sieved through a 2-mm nylon mesh screen to obtain a fine powder. In a microwave digester (ETHOS One; Milestone Inc.), $0.5 \mathrm{~g}$ dry weight (DW) of ground tissue was digested with concentrated $70 \%$ nitric acid $\left(\mathrm{HNO}_{3}\right)$ and $37 \%$ hydrochloric acid $(\mathrm{HCl})$, while $0.5 \mathrm{~g}$ of soil sample was digested with concentrated $70 \% \mathrm{HNO}_{3}$ and $30 \%$ hydrogen peroxide $\left(\mathrm{H}_{2} \mathrm{O}_{2}\right)$. The solution was diluted to $25 \mathrm{~mL}$ with $1 \% \mathrm{HNO}_{3}$ in DI water after digested samples were filtered using Whatman No. 42 filter paper. All chemicals employed in this study were TraceMetal® grade from Merck®, Germany. Depending on Cd concentration, FAAS or GF-AAS were used to examine digested plant and soil samples. A blank method and soil and plant standard reference materials (NIST SRM ${ }^{\circledR}$ 2710a Montana soil and NIST SRM® 1515 apple leaves, respectively) were used to determine the accuracy of the sample data. The percent recovery rates for the plant and soil samples were 91.4 to $109.3 \%$ and 94.2 to $109.2 \%$, respectively. The relative standard deviation (RSD) values of the plant and soil samples varied from 1.3 to $3.3 \%$ and 1.6 to $4.3 \%$, respectively. The RSD values of the samples were less than $5 \%$, showing that the method was precise.

Data and statistical analyses. Bioconcentration factor of root (BCFR) was calculated by Cd concentration of root/ext. Cd in soil ${ }^{13}$. Translocation factor (TF) was calculated by $\mathrm{Cd}$ concentration in plant shoot/Cd concentration in plant roots ${ }^{13}$. Cadmium uptake was calculated by $\mathrm{Cd}$ concentration in whole plant tissue - plant dry biomass ${ }^{14}$. The growth rate in dry biomass (GRDB) was calculated by plant dry biomass after harvest - plant dry biomass before planting/number of months of plant growth ${ }^{5}$. Root : shoot (R/S ratio) was calculated by total dry biomass of root/total dry biomass of shoots ${ }^{15}$.

Data analysis was processed using SPSS, Version 18.0. The statistical difference among treatments for each plant species and among plant species in each treatment was analyzed to obtain differences in level means using one-way analysis of variance (one-way ANOVA), followed by least significant differences (LSD) post hoc comparisons at the $95 \%$ confidence level $(p<0.05)$. All data were expressed as means \pm standard deviation (S.D.).

\section{Results And Discussion}

Physicochemical properties of amended soils Table 1 shows selected physicochemical properties of amended soils. The texture of commercial soil (C0) was loam. However, some amended soil became clay loam after being treated with various organic amendments such as SL, SLBM, SLVC, LCM, and LBM. Loam is a soil texture that contains high levels of nutrients, moisture, and humus, making it ideal for agricultural plantations ${ }^{16}$. Organic amendments were able to modestly raise the $\mathrm{pH}$ of some amended soils, e.g., SL, SBM, SLBM, LBM, BMVC, BMCM, and BMBM; pH 6-7.3, while only SLVC and LCM had a slight reduction in $\mathrm{pH}$ (5.5 and 5.8, respectively). Leonardite in SLVC, LCM, and LBM has the potential to lower soil pH, affecting plant growth and productivity. As a result, leonardite is usually mixed with other organic or inorganic amendments to achieve the desired soil pH for plant cultivation ${ }^{17}$. Electrical conductivity values in some amended soils were relatively high, exceeding the regulatory limit of $2 \mathrm{dS} \mathrm{m}^{-1}$, e.g., SL, SLVC, LCM, BMCM, and BMBM; 2.1 to $2.5 \mathrm{dS} \mathrm{m}^{-1}$. This indicated that the soils had excessively high salt levels, which could affect plant growth ${ }^{18}$. Organic amendments can raise EC contents to dangerously high levels, making it vital to maintain a note of any changes in plant symptoms that can alter the survival rates of the study plants ${ }^{19}$. When compared with $\mathrm{C} 0$ soil, the addition of various organic amendments resulted in an increase in OM of 1.3 to 1.5 times. The CEC values in some amended soils, such as SBM, BMVC, BMCM, and BMBM, decreased slightly, which could be due to the dilution effects of the organic amendments ${ }^{5}$. Higher soil pH values in some amended soils may be linked to the increased negative charge that develops on $\mathrm{OM}$ and microbial activity as a result of enhanced nutrient availability ${ }^{20}$. When compared with the identical product of commercial soil in a related study, the $\mathrm{CO}$ soil in this study had higher contents of essential nutrients (N, P, K); however, it had lower amounts of $\mathrm{Ca}$ and $\mathrm{Mg}^{15}$. Organic amendments increased essential nutrient concentrations such as total $\mathrm{N}$, Ext. P, Ext. Ca, and Ext. Mg, although Ext. $\mathrm{K}$ content was somewhat lower in practically amended soils, with the exception of T8 soils $\left(3,960 \mathrm{mg} \mathrm{kg}^{-1}\right)$ or higher than 1.4 times compared with C0 soil. 
Table 1

Physicochemical properties of the amended soils

\begin{tabular}{|c|c|c|c|c|c|c|c|c|c|c|c|}
\hline & \multirow[t]{2}{*}{ Parameter } & \multirow[t]{2}{*}{ Units } & \multicolumn{9}{|l|}{ Soil } \\
\hline & & & $\mathrm{CO}$ & SL & SBM & SLBM & SLVC & LCM & LBM & BMVC & $B N$ \\
\hline & \multicolumn{2}{|l|}{ Texture } & Loam & Clay loam & Loam & Clay loam & Clay loam & Clay loam & Clay loam & Loam & Lo \\
\hline & Sand & $\%$ & 44.8 & 42.4 & 43.4 & 42.4 & 40.2 & 39.6 & 42.2 & 44.0 & 48 \\
\hline & Silt & $\%$ & 37.6 & 28.2 & 31.2 & 28.2 & 29.2 & 32.2 & 28.2 & 30.2 & 28 \\
\hline & Clay & $\%$ & 17.6 & 29.4 & 25.4 & 29.4 & 30.6 & 28.2 & 29.6 & 25.8 & 23 \\
\hline & \multicolumn{2}{|l|}{$\mathrm{pH}$} & 5.9 & 6.6 & 7.3 & 6.9 & 5.5 & 5.8 & 6.0 & 6.8 & $6 . \mathrm{C}$ \\
\hline & EC & $\begin{array}{l}\mathrm{dS} \\
\mathrm{m}^{-1}\end{array}$ & 1.5 & 2.2 & 1.8 & 1.9 & 2.1 & 2.5 & 1.8 & 2.0 & 2.5 \\
\hline & $\mathrm{OM}$ & $\%$ & 6.8 & 9.4 & 9.0 & 8.6 & 9.9 & 9.8 & 8.9 & 9.6 & 8.5 \\
\hline & CEC & $\begin{array}{l}\mathrm{cmol}^{-1} \\
\mathrm{~kg}^{-1}\end{array}$ & 13.4 & 21.5 & 11.8 & 15.6 & 22.1 & 20.2 & 17.0 & 12.9 & 11 \\
\hline & Total N & $\%$ & 0.34 & 0.47 & 0.45 & 0.43 & 0.49 & 0.49 & 0.44 & 0.48 & 0.4 \\
\hline & Ext. P & $\begin{array}{l}\mathrm{mg} \\
\mathrm{kg}^{-1}\end{array}$ & 467 & 441 & 5928 & 4375 & 866 & 715 & 2302 & 5676 & 63 \\
\hline & Ext. $\mathrm{K}$ & $\begin{array}{l}\mathrm{mg} \\
\mathrm{kg}^{-1}\end{array}$ & 2749 & 2586 & 2478 & 2391 & 2305 & 2723 & 2085 & 2530 & 39 \\
\hline & Ext. Ca & $\begin{array}{l}\mathrm{mg} \\
\mathrm{kg}^{-1}\end{array}$ & 1375 & 3948 & 3682 & 4447 & 2832 & 2244 & 2874 & 3640 & 29 \\
\hline & Ext. Mg & $\begin{array}{l}\mathrm{mg} \\
\mathrm{kg}^{-1}\end{array}$ & 463 & 561 & 511 & 547 & 708 & 702 & 514 & 667 & 69 \\
\hline \multicolumn{12}{|c|}{ Cd concentrations measured in soils prior to planting } \\
\hline & Total Cd & $\begin{array}{l}\mathrm{mg} \\
\mathrm{kg}^{-1}\end{array}$ & BDL & BDL & BDL & BDL & $\mathrm{BDL}$ & BDL & $\mathrm{BDL}$ & BDL & $\mathrm{BD}$ \\
\hline & Ext. Cd & $\begin{array}{l}\mathrm{mg} \\
\mathrm{kg}^{-1}\end{array}$ & BDL & BDL & BDL & BDL & BDL & BDL & BDL & BDL & $\mathrm{BD}$ \\
\hline \multicolumn{12}{|c|}{ Cd concentrations measured in soils after plant harvest } \\
\hline \multirow[t]{2}{*}{ J. curcas } & Total Cd & $\begin{array}{l}\mathrm{mg} \\
\mathrm{kg}^{-1}\end{array}$ & - & $2.7 \pm 1.2 \mathrm{bA}$ & $2.9 \pm 1.2 \mathrm{bA}$ & $2.5 \pm 1.3 \mathrm{bA}$ & $2.0 \pm 0.9 \mathrm{bA}$ & $2.3 \pm 1.1 \mathrm{abA}$ & $3.1 \pm 1.1 \mathrm{bA}$ & $3.7 \pm 1.3 \mathrm{bA}$ & 3.1 \\
\hline & Ext. Cd & $\begin{array}{l}\mathrm{mg} \\
\mathrm{kg}^{-1}\end{array}$ & - & $0.7 \pm 0.2 \mathrm{bA}$ & $0.7 \pm 0.2 \mathrm{bA}$ & $0.5 \pm 0.2 \mathrm{bA}$ & $0.4 \pm 0.2 \mathrm{bA}$ & $0.7 \pm 0.2 \mathrm{abA}$ & $0.4 \pm 0.2 \mathrm{bA}$ & $0.7 \pm 0.2 \mathrm{bA}$ & 0.7 \\
\hline \multirow[t]{2}{*}{$\begin{array}{l}\text { M. } \\
\text { esculenta }\end{array}$} & Total Cd & $\begin{array}{l}\mathrm{mg} \\
\mathrm{kg}^{-1}\end{array}$ & - & $0.6 \pm 0.3 \mathrm{bcB}$ & $1.0 \pm 0.3 \mathrm{aB}$ & $0.6 \pm 0.3 \mathrm{abcB}$ & $0.4 \pm 0.2 \mathrm{cB}$ & $0.4 \pm 0.2 \mathrm{cB}$ & $0.5 \pm 0.0 \mathrm{cB}$ & $0.6 \pm 0.4 \mathrm{bcB}$ & 0.4 \\
\hline & Ext. Cd & $\begin{array}{l}\mathrm{mg} \\
\mathrm{kg}^{-1}\end{array}$ & - & $0.5 \pm 0.1 \mathrm{bcdB}$ & $0.6 \pm 0.1 \mathrm{aA}$ & $0.5 \pm 0.1 \mathrm{bcA}$ & $0.5 \pm 0.1 \mathrm{bcdA}$ & $0.4 \pm 0.1 \mathrm{deB}$ & $0.3 \pm 0.1 \mathrm{eA}$ & $0.4 \pm 0.1 \mathrm{cdeB}$ & 0.5 \\
\hline \multirow[t]{2}{*}{$\begin{array}{l}A \text {. } \\
\text { mangium }\end{array}$} & Total Cd & $\begin{array}{l}\mathrm{mg} \\
\mathrm{kg}^{-1}\end{array}$ & - & $1.5 \pm 0.9 \mathrm{bB}$ & $\begin{array}{l}2.4 \pm 0.7 \\
\mathrm{bA}\end{array}$ & $2.5 \pm 1.3 \mathrm{bA}$ & $2.3 \pm 0.3 \mathrm{bcA}$ & $2.8 \pm 0.6 \mathrm{abA}$ & $3.2 \pm 0.1 \mathrm{abA}$ & $3.5 \pm 0.3 \mathrm{aA}$ & $2 . \varepsilon$ \\
\hline & Ext. Cd & $\begin{array}{l}\mathrm{mg} \\
\mathrm{kg}^{-1}\end{array}$ & - & $0.6 \pm 0.1 \mathrm{aAB}$ & $\begin{array}{l}0.6 \pm 0.0 \\
\mathrm{aA}\end{array}$ & $0.5 \pm 0.0 \mathrm{bcA}$ & $0.4 \pm 0.1 \mathrm{cA}$ & $0.6 \pm 0.1 \mathrm{abA}$ & $0.4 \pm 0.1 \mathrm{cA}$ & $0.5 \pm 0.1 \mathrm{abcAB}$ & $0 . \epsilon$ \\
\hline
\end{tabular}

$B D L$ below detection limits, $C E C$ cation exchange capacity, EC electrical conductivity, Ext. extractable, $O M$ organic matter, $C O$ commercial soil, $S L$ soil+leonadit $S B M$ soil+bone meal (T2), SLBM soil+leonadite+bone meal (T3), SLVC soil+leonadite+earthworm manure (T4), LCM soil+leonadite+chicken manure (T5), LBI manure (T6), $B M V C$ soil+bone meal+earthworm manure (T7), BMCM soil+bone meal+chicken manure (T8), BMBM soil+bone meal+bat manure (T9)

Values followed by the same letter did not significantly differ; lower-case letters show the differences in Cd concentrations in the soils within the same plant $\varsigma$ while capital letters indicate the differences in total (or Ext.) Cd concentrations in the soils among plant species (LSD, $p<0.05)$

Before planting, total Cd and Ext. Cd concentrations in all soil samples were below detection limits (Table 1), but those values increased somewhat following plant harvest, whereas total Cd concentrations were greater than ext. Cd concentrations. High cadmium accumulation in soil has long been influenced by the $\mathrm{Cd}$ content in water and the accumulation period ${ }^{21}$. The remaining $\mathrm{Cd}$ contents in soils after the three-month harvesting period might be attributable to the addition of Cd solution to amended soils and different bioavailability rates of the study plants. When comparing all amended soils, total $\mathrm{Cd}$ and ext. Cd contents for individual plants exhibited narrow variations. Total Cd concentrations in all amended soils for jatropha, cassava, and acacia were 2 to $3.7 \mathrm{mg}$ 
$\mathrm{kg}^{-1}, 0.4$ to $1 \mathrm{mg} \mathrm{kg}^{-1}$, and 1.5 to $3.5 \mathrm{mg} \mathrm{kg}^{-1}$, respectively, whereas ext. Cd concentrations for jatropha, cassava, and acacia were 0.4 to $0.7 \mathrm{mg} \mathrm{kg}{ }^{-1}, 0.3$ to $0.6 \mathrm{mg} \mathrm{kg}^{-1}$, and 0.4 to $0.6 \mathrm{mg} \mathrm{kg}^{-1}$, respectively.

Effects of amendments on plant growth. In this study, jatropha and acacia thrived in Cd soils for three months, with no visible phytotoxic effects in any of the treatments. Cassava also had a 100\% survival rate; however, this crop plant showed symptoms of stress such as deformed leaves and yellowing in almost all treatments. During the study period, however, Cassava cultivated in T1 treatment showed no signs of harm. Of three crop plants surveyed, only Cassava was found to be cultivated in $\mathrm{Cd}$ and $\mathrm{Zn}$ co-contaminated agricultural areas of the Mae Tao River Basin. However, no research publications are available on all of the study plants growing in those locations.

The use of soil amendment had variable effects on plant growth (plant height, root length, and dry biomass production). Furthermore, all of the study plants had considerably improved plant height and root length, as well as enhanced dry biomass production, from planting to harvesting periods (Table 2). Except for jatropha, the GRDB values of the study plants also revealed similar trends to those of plant growth performances, with GRDB values in month 3 slightly lower than month 1. The following is the descending order of growth rate in dry biomass values for the study plants: cassava > acacia > jatropha. 
Table 2

Growth performances of the study plants in a pot study for 3 months $(n=4)$

\begin{tabular}{|c|c|c|c|c|c|c|}
\hline Scientific name & Treatment & Month & Height (cm) & Root (cm) & Biomass (g plant ${ }^{-1}$ ) & Growth rate in dry biomass \\
\hline \multirow[t]{27}{*}{ J. curcas } & $\mathrm{T} 1$ & 0 & $36.7 \pm 0.6 a A^{2}$ & $0.0 \pm 0.0 \mathrm{aB}^{3}$ & $11.0 \pm 1.7 a A^{3}$ & - \\
\hline & & 1 & $51.3 \pm 2.1 \mathrm{aB}^{1}$ & $14.5 \pm 1.1 \mathrm{aC}^{2}$ & $27.8 \pm 2.3 a A^{2}$ & $17.4 \pm 2.3 a A^{1}$ \\
\hline & & 3 & $50.8 \pm 5.0 \mathrm{bA}{ }^{1}$ & $20.3 \pm 2.7 b c^{1}$ & $59.9 \pm 7.4 a b A^{1}$ & $16.3 \pm 2.5 \mathrm{abA}^{1}$ \\
\hline & $\mathrm{T} 2$ & 0 & $36.7 \pm 0.6 a A^{2}$ & $0.0 \pm 0.0 \mathrm{aB}^{3}$ & $11.0 \pm 1.7 a A^{3}$ & - \\
\hline & & 1 & $43.7 \pm 4.7 a b c B^{2}$ & $11.4 \pm 5.1 \mathrm{abB}^{2}$ & $33.0 \pm 5.9 a A^{2}$ & $22.5 \pm 5.9 a A^{1}$ \\
\hline & & 3 & $66.1 \pm 10.5 \mathrm{a} \mathrm{A}^{1}$ & $24.5 \pm 4.1 \mathrm{abA}^{1}$ & $62.5 \pm 2.5 \mathrm{a} \mathrm{A}^{1}$ & $17.2 \pm 0.8 \mathrm{a} \mathrm{A}^{1}$ \\
\hline & T3 & 0 & $36.7 \pm 0.6 a A^{2}$ & $0.0 \pm 0.0 \mathrm{aB}^{3}$ & $11.0 \pm 1.7 a A^{3}$ & - \\
\hline & & 1 & $38.8 \pm 4.3 c B^{2}$ & $12.1 \pm 6.6 a b B^{2}$ & $25.7 \pm 5.6 a A^{2}$ & $15.3 \pm 5.6 a A^{1}$ \\
\hline & & 3 & $60.8 \pm 2.3 a b A^{1}$ & $29.6 \pm 11.3 a A^{1}$ & $52.4 \pm 8.7 a b c A^{1}$ & $13.8 \pm 2.9 a b c A^{1}$ \\
\hline & $\mathrm{T} 4$ & 0 & $36.7 \pm 0.6 \mathrm{a} \mathrm{A}^{2}$ & $0.0 \pm 0.0 \mathrm{aB}^{2}$ & $11.0 \pm 1.7 a A^{2}$ & - \\
\hline & & 1 & $47.6 \pm 11.7 a b B^{1,2}$ & $10.6 \pm 5.7 a b B^{1}$ & $27.2 \pm 12.4 a A^{1,2}$ & $16.8 \pm 12.4 a \mathrm{~A}^{1}$ \\
\hline & & 3 & $55.2 \pm 13.4 \mathrm{abA}^{1}$ & $19.3 \pm 1.7 b B^{1}$ & $42.4 \pm 16.0 \mathrm{bcB}{ }^{1}$ & $10.5 \pm 5.3 \mathrm{bcB} \mathrm{B}^{1}$ \\
\hline & T5 & 0 & $36.7 \pm 0.6 a A^{2}$ & $0.0 \pm 0.0 \mathrm{aB}^{3}$ & $11.0 \pm 1.7 a A^{2}$ & - \\
\hline & & 1 & $38.2 \pm 6.7 c A^{2}$ & $7.1 \pm 1.6 b_{c B}{ }^{2}$ & $14.3 \pm 4.0 \mathrm{bA}^{2}$ & $3.8 \pm 4.0 \mathrm{bA}^{1}$ \\
\hline & & 3 & $58.9 \pm 13.6 a b A^{1}$ & $16.3 \pm 5.6 b^{1}$ & $46.8 \pm 21.1 \mathrm{abcA}^{1}$ & $11.9 \pm 7.0 \mathrm{abcA}^{1}$ \\
\hline & T6 & 0 & $36.7 \pm 0.6 a A^{3}$ & $0.0 \pm 0.0 \mathrm{aB}^{3}$ & $11.0 \pm 1.7 a A^{3}$ & - \\
\hline & & 1 & $45.3 \pm 1.9 \mathrm{abcB}{ }^{2}$ & $11.0 \pm 1.7 a b B^{2}$ & $25.6 \pm 3.6 a A^{2}$ & $15.1 \pm 3.6 \mathrm{a} \mathrm{A}^{1}$ \\
\hline & & 3 & $58.0 \pm 8.9 a^{1} B^{1}$ & $22.5 \pm 1.7 a b A B^{1}$ & $57.4 \pm 13.3 \mathrm{abA} A^{1}$ & $15.5 \pm 4.4 \mathrm{abA}^{1}$ \\
\hline & T7 & 0 & $36.7 \pm 0.6 a A^{1}$ & $0.0 \pm 0.0 \mathrm{aB}^{3}$ & $11.0 \pm 1.7 a A^{2}$ & - \\
\hline & & 1 & $41.7 \pm 3.3 b c B^{1}$ & $6.2 \pm 5.0 \mathrm{bcB}^{2}$ & $28.9 \pm 7.6 \mathrm{a} \mathrm{A}^{1}$ & $18.6 \pm 7.7 a A^{1}$ \\
\hline & & 3 & $50.3 \pm 14.8 \mathrm{bA}^{1}$ & $20.5 \pm 5.9 b A^{1}$ & $42.1 \pm 15.0 b_{c} A^{1}$ & $10.4 \pm 5.0 \mathrm{bcA}^{1}$ \\
\hline & T8 & 0 & $36.7 \pm 0.6 a A^{2}$ & $0.0 \pm 0.0 \mathrm{aB}^{3}$ & $11.0 \pm 1.7 a A^{3}$ & - \\
\hline & & 1 & $38.4 \pm 3.0 \mathrm{cA}^{2}$ & $3.5 \pm 1.8 \mathrm{cB}^{2}$ & $23.1 \pm 7.2 \mathrm{abA}^{2}$ & $12.7 \pm 7.2 \mathrm{abA}^{1}$ \\
\hline & & 3 & $53.1 \pm 12.4 \mathrm{abAB}^{1}$ & $24.1 \pm 3.0 a A^{1}$ & $39.0 \pm 10.0 \mathrm{cAB} \mathrm{B}^{1}$ & $9.4 \pm 3.3 c A^{1}$ \\
\hline & T9 & 0 & $36.7 \pm 0.6 a A^{3}$ & $0.0 \pm 0.0 \mathrm{aB}^{3}$ & $11.0 \pm 1.7 a A^{3}$ & - \\
\hline & & 1 & $47.0 \pm 2.7 a b A^{2}$ & $11.9 \pm 5.1 \mathrm{abB}^{2}$ & $23.6 \pm 9.6 a b A^{2}$ & $13.1 \pm 9.6 a A^{1}$ \\
\hline & & 3 & $61.8 \pm 1.3 a b A^{1}$ & $23.6 \pm 2.5 a b A^{1}$ & $53.3 \pm 9.6 a b c B^{1}$ & $14.1 \pm 3.2 \mathrm{abcB}^{1}$ \\
\hline \multirow[t]{7}{*}{ M. esculenta } & $\mathrm{T} 1$ & 0 & $35.7 \pm 0.7 a A^{2}$ & $0.0 \pm 0.0 \mathrm{aB}^{2}$ & $8.3 \pm 1.4 \mathrm{aB}^{2}$ & - \\
\hline & & 1 & $61.1 \pm 5.0 \mathrm{aA} \mathrm{A}^{1}$ & $22.8 \pm 3.2 a^{a b B}{ }^{1}$ & $19.5 \pm 7.4 \mathrm{aB}^{2}$ & $11.2 \pm 7.4 a A^{1}$ \\
\hline & & 3 & $60.8 \pm 1.9 \mathrm{bA}^{1}$ & $27.3 \pm 5.1 \mathrm{abcdB}^{1}$ & $56.7 \pm 17.8 \mathrm{a} \mathrm{A}^{1}$ & $16.1 \pm 5.9 a A^{1}$ \\
\hline & $\mathrm{T} 2$ & 0 & $35.7 \pm 0.7 a A^{2}$ & $0.0 \pm 0.0 \mathrm{aB}^{3}$ & $8.3 \pm 1.4 \mathrm{aB}^{2}$ & - \\
\hline & & 1 & $62.0 \pm 7.2 \mathrm{a} \mathrm{A}^{1}$ & $17.0 \pm 8.7 a b c A B^{2}$ & $21.7 \pm 3.5 \mathrm{aB}^{2}$ & $13.4 \pm 3.5 \mathrm{aB}^{1}$ \\
\hline & & 3 & $66.5 \pm 8.7 a A^{1}$ & $33.8 \pm 6.3 a b A^{1}$ & $40.7 \pm 22.7 a A^{1}$ & $14.9 \pm 7.5 \mathrm{a} \mathrm{A}^{1}$ \\
\hline & T3 & 0 & $35.7 \pm 0.7 a A^{3}$ & $0.0 \pm 0.0 \mathrm{aB}^{3}$ & $8.3 \pm 1.4 \mathrm{aB}^{2}$ & - \\
\hline \multicolumn{7}{|c|}{$\begin{array}{l}\text { Values followed by the same letter did not significantly differ; lower case letters indicate the differences in growth performance among treatments within } \\
\text { the same plant species and growth period (LSD, } p<0.05) \text {; capital letters indicate the difference in growth performance among plant species within the } \\
\text { same treatment (LSD: } p<0.05) \text {; and numbers indicate the difference in growth performance among growth periods within the same plant species and } \\
\text { treatment. }\end{array}$} \\
\hline
\end{tabular}




\begin{tabular}{|c|c|c|c|c|c|c|}
\hline & & 1 & $58.1 \pm 3.5 a b A^{2}$ & $13.6 \pm 6.3 \mathrm{cB}^{2}$ & $21.1 \pm 8.48 a \mathrm{AB}^{2}$ & $12.8 \pm 8.5 a A^{1}$ \\
\hline & & 3 & $67.5 \pm 5.1 a^{a b A^{1}}$ & $36.3 \pm 13.2 \mathrm{a} \mathrm{A}^{1}$ & $51.0 \pm 22.9 a A^{1}$ & $14.2 \pm 7.6 \mathrm{aA}^{1}$ \\
\hline & $\mathrm{T} 4$ & 0 & $35.7 \pm 0.7 a A^{2}$ & $0.0 \pm 0.0 \mathrm{aB}^{2}$ & $8.3 \pm 1.4 \mathrm{aB}^{2}$ & - \\
\hline & & 1 & $65.2 \pm 8.2 \mathrm{a} \mathrm{A}^{1}$ & $22.9 \pm 1.9 a b b^{1}$ & $15.0 \pm 5.9 a A B^{2}$ & $6.7 \pm 5.9 \mathrm{a} \mathrm{A}^{2}$ \\
\hline & & 3 & $71.8 \pm 2.6 \mathrm{a} \mathrm{A}^{1}$ & $28.1 \pm 6.4 a b c d A B^{1}$ & $48.0 \pm 15.8 \mathrm{a} \mathrm{A}^{1}$ & $18.8 \pm 4.5 a \mathrm{~A}^{1}$ \\
\hline & T5 & 0 & $35.7 \pm 0.7 a A^{3}$ & $0.0 \pm 0.0 \mathrm{aB}^{2}$ & $8.3 \pm 1.4 \mathrm{aB}^{2}$ & - \\
\hline & & 1 & $43.9 \pm 6.3 c A^{2}$ & $15.1 \pm 8.1 b^{\prime} B^{1}$ & $13.0 \pm 4.8 \mathrm{a} \mathrm{A}^{2}$ & $4.6 \pm 4.8 \mathrm{a} \mathrm{A}^{2}$ \\
\hline & & 3 & $70.3 \pm 5.3 a A^{1}$ & $17.6 \pm 1.5 \mathrm{~dB}^{1}$ & $64.4 \pm 7.5 \mathrm{a} \mathrm{A}^{1}$ & $18.7 \pm 2.5 a A^{1}$ \\
\hline & T6 & 0 & $35.7 \pm 0.7 a A^{3}$ & $0.0 \pm 0.0 \mathrm{aB}^{2}$ & $8.3 \pm 1.4 \mathrm{aB}^{2}$ & - \\
\hline & & 1 & $64.9 \pm 4.6 a A^{2}$ & $25.0 \pm 6.3 a A^{1}$ & $19.4 \pm 8.4 a A B^{2}$ & $11.1 \pm 8.4 \mathrm{aA}^{1}$ \\
\hline & & 3 & $73.1 \pm 4.9 \mathrm{a} \mathrm{A}^{1}$ & $19.8 \pm 4.1 \mathrm{cdB}^{1}$ & $75.1 \pm 41.6 a A^{1}$ & $22.2 \pm 13.9 a A^{1}$ \\
\hline & T7 & 0 & $35.7 \pm 0.7 a A^{2}$ & $0.0 \pm 0.0 \mathrm{aB}^{2}$ & $8.3 \pm 1.4 \mathrm{aB}^{2}$ & - \\
\hline & & 1 & $58.8 \pm 1.7 a b A^{1}$ & $20.3 \pm 9.3 a b c A^{1}$ & $15.8 \pm 4.6 \mathrm{aB}^{2}$ & $7.5 \pm 4.6 \mathrm{aB}^{2}$ \\
\hline & & 3 & $64.1 \pm 8.8 \mathrm{abA}^{1}$ & $29.4 \pm 10.6 a b c d A^{1}$ & $41.9 \pm 7.3 a A^{1}$ & $16.0 \pm 3.9 a A^{1}$ \\
\hline & T8 & 0 & $35.7 \pm 0.7 a A^{3}$ & $0.0 \pm 0.0 \mathrm{aB}^{2}$ & $8.3 \pm 1.4 \mathrm{aB}^{2}$ & - \\
\hline & & 1 & $50.2 \pm 11.4 b_{c} A^{2}$ & $14.5 \pm 3.1 \mathrm{bcA}^{1}$ & $17.5 \pm 7.0 \mathrm{a} \mathrm{A}^{2}$ & $9.2 \pm 7.0 \mathrm{aA}^{1}$ \\
\hline & & 3 & $65.0 \pm 8.8 a b A^{1}$ & $21.9 \pm 4.3 \mathrm{bcd} \mathrm{A}^{1}$ & $36.6 \pm 17.0 \mathrm{aA}{ }^{1}$ & $17.3 \pm 9.9 a A^{1}$ \\
\hline & T9 & 0 & $35.7 \pm 0.7 a A^{3}$ & $0.0 \pm 0.0 \mathrm{aB}^{2}$ & $8.3 \pm 1.4 \mathrm{aB}^{2}$ & - \\
\hline & & 1 & $45.3 \pm 8.4 c A^{2}$ & $13.4 \pm 2.1 \mathrm{cB}^{1}$ & $15.2 \pm 3.3 a A^{2}$ & $6.9 \pm 3.3 \mathrm{a} \mathrm{A}^{2}$ \\
\hline & & 3 & $61.1 \pm 5.5 \mathrm{bA}^{1}$ & $31.5 \pm 19.2 \mathrm{abcA}^{1}$ & $45.0 \pm 18.8 \mathrm{a} \mathrm{A}^{1}$ & $22.5 \pm 3.9 a A^{1}$ \\
\hline A. mangium & $\mathrm{T} 1$ & 0 & $17.4 \pm 1.7 \mathrm{aB}^{3}$ & $12.9 \pm 3.0 a A^{2}$ & $3.6 \pm 0.4 \mathrm{aC}^{2}$ & - \\
\hline & & 1 & $28.3 \pm 4.8 \mathrm{abcdC}^{2}$ & $34.5 \pm 6.0 \mathrm{a} \mathrm{A}^{1}$ & $9.6 \pm 3.4 b c^{2}$ & $6.1 \pm 3.4 \mathrm{bB}^{2}$ \\
\hline & & 3 & $56.7 \pm 10.3 \mathrm{abcdA}^{1}$ & $40.8 \pm 4.1 \mathrm{aA}^{1}$ & $17.8 \pm 6.8 \mathrm{bcdB}{ }^{1}$ & $14.3 \pm 6.8 \mathrm{bcdA}^{1}$ \\
\hline & T2 & 0 & $17.4 \pm 1.7 \mathrm{aB}^{3}$ & $12.9 \pm 3.0 \mathrm{aA}^{2}$ & $3.6 \pm 0.4 \mathrm{aC}^{2}$ & - \\
\hline & & 1 & $34.5 \pm 6.0 a^{a b B^{2}}$ & $25.8 \pm 7.6 a b A^{1}$ & $10.7 \pm 2.6 b c^{2}$ & $7.1 \pm 2.6 \mathrm{bB}^{2}$ \\
\hline & & 3 & $68.9 \pm 12.9 \mathrm{abA}^{1}$ & $29.8 \pm 7.8 a b c A^{1}$ & $23.0 \pm 9.0 a^{a b B}{ }^{1}$ & $19.4 \pm 9.0 a^{b} A^{1}$ \\
\hline & T3 & 0 & $17.4 \pm 1.7 \mathrm{aB}^{2}$ & $12.9 \pm 3.0 a A^{2}$ & $3.6 \pm 0.4 \mathrm{aC}^{2}$ & - \\
\hline & & 1 & $37.1 \pm 12.2 \mathrm{aB}^{2}$ & $29.2 \pm 11.1 \mathrm{abA}^{1}$ & $14.0 \pm 4.8 \mathrm{abB}^{1}$ & $10.5 \pm 4.9 \mathrm{abA}^{1}$ \\
\hline & & 3 & $74.1 \pm 26.3 \mathrm{a} \mathrm{A}^{1}$ & $33.0 \pm 11.6 a b c A^{1}$ & $9.6 \pm 5.36 \mathrm{cdB}^{1,2}$ & $6.1 \pm 5.4 \mathrm{cdA}^{1,2}$ \\
\hline & $\mathrm{T} 4$ & 0 & $17.4 \pm 1.7 \mathrm{aB}^{2}$ & $12.9 \pm 3.0 a^{2}$ & $3.6 \pm 0.4 a C^{2}$ & - \\
\hline & & 1 & $28.9 \pm 5.9 a b c d C^{2}$ & $31.6 \pm 8.9 a b c d A^{1}$ & $10.8 \pm 4.0 \mathrm{bB}^{1}$ & $7.3 \pm 4.0 \mathrm{bA}^{1}$ \\
\hline & & 3 & $57.8 \pm 12.8 a b c d A^{1}$ & $36.6 \pm 7.0 \mathrm{abA} \mathrm{A}^{1}$ & $8.6 \pm 3.7 d c^{1}$ & $5.1 \pm 3.7 \mathrm{~dB}^{1}$ \\
\hline & T5 & 0 & $17.4 \pm 1.7 \mathrm{aB}^{2}$ & $12.9 \pm 3.0 a^{2}$ & $3.6 \pm 0.4 a C^{1}$ & - \\
\hline & & 1 & $18.6 \pm 1.4 \mathrm{~dB}^{2}$ & $26.6 \pm 6.0 \mathrm{dA}^{1}$ & $10.4 \pm 3.0 \mathrm{bA}{ }^{1}$ & $6.9 \pm 3.0 \mathrm{bA}^{1}$ \\
\hline & & 3 & $37.1 \pm 3.0 \mathrm{~dB}^{1}$ & $32.6 \pm 6.1 \mathrm{abcA}^{1}$ & $10.9 \pm 9.4 \mathrm{cdB}^{1}$ & $9.8 \pm 9.7 b c d A^{1}$ \\
\hline & T6 & 0 & $17.4 \pm 1.7 \mathrm{aB}^{2}$ & $12.9 \pm 3.0 \mathrm{a} \mathrm{A}^{2}$ & $3.6 \pm 0.4 a \mathrm{C}^{2}$ & - \\
\hline
\end{tabular}




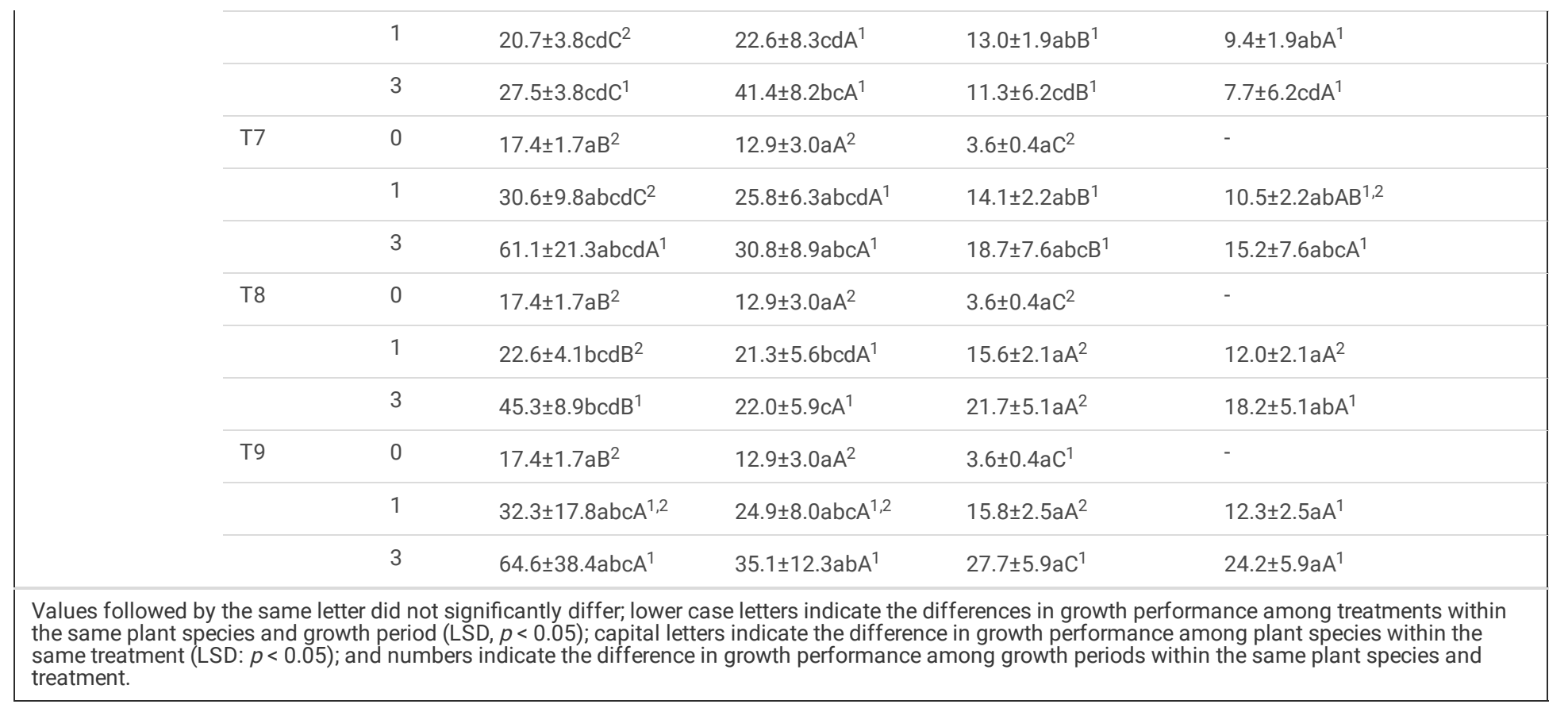

After harvest, BMBM in T9 treatment promoted the best growth performance in acacia (dry biomass $27.7 \mathrm{~g} \mathrm{plant}^{-1}$, GRDB 24.2 ), followed by LBM in T6 treatment for Cassava (dry biomass $75.1 \mathrm{~g} \mathrm{plant}^{-1}$, GRDB 22.2). Bone meal and bat manure are more widely used as a $\mathrm{P}$ source in commercial fertilizer because of their high $\mathrm{P}$ content ( 7 to $12 \%$ and 1 to $9 \%$, respectively). Bone meal has an NPK of 3-15-0 on average, making it a rich source of $\mathrm{P}$ for plants. Bat manure, commonly known as "Guano," is an important organic source of nitrogen that also contains minerals like struvite and magnesium, making it useful for fertilizing crops to some extent ${ }^{22}$. Leonardite is an end-product of lignite coal and mined pits at Mae Moh mine in Lampang Province, which has a high total $\mathrm{N}$ concentration ( $0.6 \%)$ but low $\mathrm{Cd}$ and $\mathrm{Zn}$ contents $\left(0.7 \mathrm{mg} \mathrm{Cd} \mathrm{kg}^{-1} \text { and } 40 \mathrm{mg} \mathrm{Zn} \mathrm{kg}^{-1} \text {, respectively }\right)^{1}$. It has been employed as organic amendment mixtures to promote crop plants, particularly Thai commercial rice cultivars, indicating that it provides a highly effective soil amendment ${ }^{5}$. In this study, the treatment that received both bone meal and bat manure supplements had 1.2 times higher dry biomass after harvest than treatment that just received bone meal. Some research also indicated that a combination of amendment supplements could increase extractable essential micronutrients and soil enzymatic activity ${ }^{23}$. However, jatropha preferred bone meal alone, as shown by the T2 treatment having the highest biomass and GRDB compared with the other treatments $\left(p<0.05 ; 62.5\right.$ g plant $^{-1}$, GRDB 17.2).

The other treatments (T3, T4, T5, T7, and T8) exhibited lower growth performances, particularly in jatropha and acacia, indicating that a specialized soil amendment, such as bat, bone meal/or leonardite alone, would be more beneficial. The combination of chicken manure or vermicompost with leonardite or bone meal was unable to support the growth of the study plants until they had attained substantial values of dry biomass production and GRDB (Table 2). In Thailand's agricultural areas, vermicompost and chicken manure are important soil organic amendments. They were reported to be successful in replacing chemical fertilizer and stimulating plant growth when combined with other organic amendments or materials, e.g., rice husk charcoal ${ }^{24}$. Because numerous research studies have suggested that chicken manure alone at high concentration may achieve targeted plant growth, the lower growth performance of the study plants might have been due to insufficient amounts of the organic amendment ${ }^{25,26}$. Furthermore, the growth performances of Cassava did not differ significantly across treatments after harvest $(p>0.05)$. This means that the combination of chicken manure or vermicompost with leonardite or bone meal as soil organic amendments for cassava is a suitable alternative amendment for planting.

The results clearly demonstrated that jatropha and acacia had lower R/S ratios than cassava (Fig. 1). The R/S ratios of the study plants were observed in ascending order as follows: jatropha (0.04 to 0.06$)>$ acacia $(0.16$ to 0.8$)>$ cassava (0.59 to 3). According to Meeinkuirt et al. ${ }^{27}$ (2013), a high R/S ratio value indicated $\mathrm{Cd}$ toxicity in plants, and so increasing $\mathrm{Cd}$ concentration in plant media is thought to harm the study plants. Substantial root/shoot ratio values were seen in all treatments for Cassava, particularly in T5 and T6 treatments, which might be linked to increased plant stress ( 3 and 2.63 , respectively).

Cadmium concentrations in plant tissues. Because soil amendments had varied impacts on different plant tissues, amended soil treatments in this study revealed varying levels of $\mathrm{Cd}$ accumulation and uptake depending on the crop plant species (Table 3). Furthermore, the study plants accumulated Cd primarily in their roots after one month of growth, with average values of 2.4 to $6.2 \mathrm{mg} \mathrm{kg}^{-1}, 4$ to $15 \mathrm{mg} \mathrm{kg}^{-1}$, and 3.3 to $4 \mathrm{mg} \mathrm{kg}^{-1}$ for jatropha, cassava, and acacia, respectively. T5 treatment resulted in the maximum Cd accumulation in cassava $\left(15 \mathrm{mg} \mathrm{kg}^{-1}\right)$, whereas T2 treatment resulted in the lowest value for acacia $\left(3.3 \mathrm{mg} \mathrm{kg}^{-1}\right)$. 
Table 3

Cd accumulation and uptake, bioconcentration factor for root (BCFR) and translocation factor (TF) among the study plants in a pot stu

\begin{tabular}{|c|c|c|c|c|c|c|c|c|c|c|}
\hline \multirow{2}{*}{$\begin{array}{l}\text { Scientific } \\
\text { name }\end{array}$} & \multirow[t]{2}{*}{ Treatment } & \multirow[t]{2}{*}{ Month } & \multicolumn{6}{|c|}{ Cd accumulation in plants $\left(\mathrm{mg} \mathrm{kg}^{-1}\right)$} & \multirow{2}{*}{$\begin{array}{l}\text { Cd uptake (mg } \\
\text { plant }^{-1} \text { ) }\end{array}$} & \multirow[t]{2}{*}{ B } \\
\hline & & & Leaf & Stem & Root & $\begin{array}{l}\text { Cassava } \\
\text { tuber }\end{array}$ & Seed & Whole plant & & \\
\hline \multirow[t]{27}{*}{ J. curcas } & T1 & 0 & $\mathrm{BDL}$ & $\mathrm{BDL}$ & $\mathrm{BDL}$ & - & - & $\mathrm{BDL}$ & $\mathrm{BDL}$ & - \\
\hline & & 1 & $3.3 \pm 1.0 \mathrm{a} \mathrm{A}^{2}$ & $3.4 \pm 1.2 \mathrm{a} \mathrm{A}^{1}$ & $5.9 \pm 1.5 \mathrm{a} \mathrm{A}^{1}$ & - & - & $4.2 \pm 0.6 \mathrm{a} \mathrm{A}^{1}$ & $120.1 \pm 20.4 a A^{2}$ & 6 \\
\hline & & 3 & $4.4 \pm 0.8 \mathrm{bA}^{1}$ & $2.3 \pm 0.8 b c B^{2}$ & $3.2 \pm 2.2 a \mathrm{bA}^{2}$ & - & - & $3.3 \pm 0.8 a A B^{2}$ & $197.0 \pm 22.8 a b A^{1}$ & 1 \\
\hline & T2 & 0 & $\mathrm{BDL}$ & $\mathrm{BDL}$ & $\mathrm{BDL}$ & - & - & $\mathrm{BDL}$ & BDL & - \\
\hline & & 1 & $3.3 \pm 1.0 \mathrm{a} \mathrm{A}^{2}$ & $3.1 \pm 1.2 \mathrm{abA}^{1}$ & $4.0 \pm 1.1 \mathrm{cA}^{1}$ & - & - & $3.5 \pm 0.5 b c A^{1}$ & $116.2 \pm 21.7 a b A^{2}$ & 3 \\
\hline & & 3 & $4.4 \pm 0.8 \mathrm{bA} \mathrm{A}^{1}$ & $2.5 \pm 0.7 \mathrm{abcA} \mathrm{A}^{2}$ & $3.5 \pm 1.6 a b A^{1}$ & - & $0.6 \pm 0.3 a$ & $3.5 \pm 0.6 \mathrm{a} \mathrm{A}^{1}$ & $217.0 \pm 13.1 \mathrm{aA} \mathrm{A}^{1}$ & 1 \\
\hline & T3 & 0 & $\mathrm{BDL}$ & $\mathrm{BDL}$ & $\mathrm{BDL}$ & - & - & $\mathrm{BDL}$ & BDL & - \\
\hline & & 1 & $2.8 \pm 1.0 \mathrm{a} \mathrm{A}^{2}$ & $2.9 \pm 1.1 \mathrm{bA}^{1}$ & $3.9 \pm 1.9 \mathrm{CAB}^{1}$ & - & - & $3.0 \pm 0.8 \mathrm{cdAB}{ }^{2}$ & $79.1 \pm 17.7 a b c A^{2}$ & 9 \\
\hline & & 3 & $4.8 \pm 1.0 \mathrm{abA}{ }^{1}$ & $2.2 \pm 0.7 \mathrm{cB}^{2}$ & $3.2 \pm 1.7 a b A^{1}$ & - & - & $3.4 \pm 0.8 \mathrm{a} \mathrm{A}^{1}$ & $177.8 \pm 32.4 a b A^{1}$ & 1 \\
\hline & T4 & 0 & $\mathrm{BDL}$ & $\mathrm{BDL}$ & $\mathrm{BDL}$ & - & - & BDL & $\mathrm{BDL}$ & - \\
\hline & & 1 & $2.9 \pm 1.1 \mathrm{a} \mathrm{A}^{2}$ & $2.9 \pm 1.1 \mathrm{bA}^{1}$ & $6.0 \pm 1.3 \mathrm{a} \mathrm{A}^{1}$ & - & - & $3.6 \pm 0.9 \mathrm{abcA}^{1}$ & $102.4 \pm 53.3 a b A^{1}$ & 1 \\
\hline & & 3 & $4.2 \pm 0.8 \mathrm{bA} \mathrm{A}^{1}$ & $2.6 \pm 0.9 a b c A^{1}$ & $3.9 \pm 1.9 \mathrm{aB}^{2}$ & - & $0.5 \pm 0.1 a$ & $3.3 \pm 1.0 \mathrm{aAB}{ }^{1}$ & $147.6 \pm 74.5 a b A^{1}$ & 2 \\
\hline & T5 & 0 & $\mathrm{BDL}$ & $\mathrm{BDL}$ & $\mathrm{BDL}$ & - & - & $\mathrm{BDL}$ & $\mathrm{BDL}$ & - \\
\hline & & 1 & $3.3 \pm 1.3 \mathrm{a} \mathrm{A}^{1}$ & $3.2 \pm 0.8 a b A^{1}$ & $5.0 \pm 1.0 \mathrm{bB}^{1}$ & - & - & $3.4 \pm 0.8 a b A^{1}$ & $54.8 \pm 12.0 \mathrm{cAB}^{2}$ & 3 \\
\hline & & 3 & $4.4 \pm 1.0 \mathrm{bA}^{1}$ & $2.6 \pm 0.8 a b c A^{1}$ & $2.2 \pm 1.7 b^{2}$ & - & - & $3.0 \pm 0.9 \mathrm{a} \mathrm{A}^{1}$ & $145.2 \pm 68.8 \mathrm{abA}^{1}$ & 1 \\
\hline & T6 & 0 & $\mathrm{BDL}$ & $\mathrm{BDL}$ & $\mathrm{BDL}$ & - & - & $\mathrm{BDL}$ & $\mathrm{BDL}$ & - \\
\hline & & 1 & $3.0 \pm 1.0 \mathrm{a} \mathrm{A}^{2}$ & $2.9 \pm 1.2 b A^{1}$ & $2.4 \pm 0.5 \mathrm{~dB}^{1}$ & - & - & $2.7 \pm 0.4 \mathrm{~dB}^{2}$ & $71.3 \pm 11.3 b_{c} A^{2}$ & 1 \\
\hline & & 3 & $4.6 \pm 0.8 a b A^{1}$ & $2.5 \pm 0.9 \mathrm{abcA}^{1}$ & $2.9 \pm 1.7 a b B^{1}$ & - & - & $3.4 \pm 0.5 \mathrm{a} \mathrm{A}^{1}$ & $193.3 \pm 46.8 a A^{1}$ & 1 \\
\hline & T7 & 0 & $\mathrm{BDL}$ & $\mathrm{BDL}$ & $\mathrm{BDL}$ & - & - & BDL & $\mathrm{BDL}$ & - \\
\hline & & 1 & $3.2 \pm 1.1 \mathrm{aA}^{2}$ & $2.9 \pm 0.8 \mathrm{bA} \mathrm{A}^{1}$ & $5.1 \pm 0.9 \mathrm{bA}^{1}$ & - & - & $3.2 \pm 0.8 \mathrm{abA}{ }^{1}$ & $109.5 \pm 24.1 \mathrm{abA}^{1}$ & 4 \\
\hline & & 3 & $5.1 \pm 1.2 \mathrm{aA}^{1}$ & $2.7 \pm 0.7 a b A^{1}$ & $3.4 \pm 1.7 a b B^{2}$ & - & - & $3.5 \pm 0.8 \mathrm{a} A \mathrm{~B}^{1}$ & $151.9 \pm 68.4 \mathrm{abA}^{1}$ & 0 \\
\hline & T8 & 0 & $\mathrm{BDL}$ & $\mathrm{BDL}$ & $\mathrm{BDL}$ & - & - & $\mathrm{BDL}$ & $\mathrm{BDL}$ & - \\
\hline & & 1 & $3.1 \pm 0.9 a A^{2}$ & $3.1 \pm 1.3 a b A^{1}$ & $6.2 \pm 1.8 \mathrm{aA} \mathrm{A}^{1}$ & - & - & $3.5 \pm 1.3 a A^{1}$ & $101.4 \pm 47.4 \mathrm{abA}^{1}$ & 7 \\
\hline & & 3 & $4.5 \pm 0.8 a b A^{1}$ & $2.7 \pm 0.9 a b c A^{1}$ & $2.9 \pm 2.1 \mathrm{abA}^{2}$ & - & - & $3.2 \pm 1.0 \mathrm{a} \mathrm{A}^{1}$ & $127.8 \pm 60.3 b A^{1}$ & 1 \\
\hline & T9 & 0 & $\mathrm{BDL}$ & $\mathrm{BDL}$ & $\mathrm{BDL}$ & - & - & BDL & BDL & - \\
\hline & & 1 & $2.9 \pm 1.0 \mathrm{aA} \mathrm{A}^{2}$ & $3.0 \pm 1.0 a b A^{1}$ & $4.9 \pm 1.2 b^{2}$ & - & - & $3.4 \pm 0.8 \mathrm{bcA}^{1}$ & $83.8 \pm 41.9 a b c A^{2}$ & 6 \\
\hline & & 3 & $4.6 \pm 0.9 \mathrm{abA} \mathrm{A}^{1}$ & $2.9 \pm 0.8 \mathrm{aAB}{ }^{1}$ & $3.1 \pm 1.6 a b B^{2}$ & - & - & $3.5 \pm 0.7 \mathrm{a} \mathrm{A}^{1}$ & $187.9 \pm 32.9 \mathrm{abA}^{1}$ & 1 \\
\hline \multirow{7}{*}{$\begin{array}{l}\text { M. } \\
\text { esculenta }\end{array}$} & $\mathrm{T} 1$ & 0 & $\mathrm{BDL}$ & $\mathrm{BDL}$ & $\mathrm{BDL}$ & - & - & $\mathrm{BDL}$ & $\mathrm{BDL}$ & - \\
\hline & & 1 & $2.5 \pm 0.9 a b B^{1}$ & $1.8 \pm 1.1 \mathrm{dC}^{2}$ & $5.7 \pm 2.4 \mathrm{bA}^{1}$ & - & - & $3.3 \pm 0.6 \mathrm{bB}^{1}$ & $53.6 \pm 18.2 \mathrm{aB}^{2}$ & 2 \\
\hline & & 3 & $1.8 \pm 0.5 \mathrm{cdC}^{2}$ & $3.1 \pm 0.5 \mathrm{a} \mathrm{A}^{1}$ & $3.9 \pm 1.4 \mathrm{abA}^{2}$ & - & - & $2.9 \pm 0.6 b c B^{1}$ & $154.7 \pm 51.7 \mathrm{a} \mathrm{A}^{1}$ & 1 \\
\hline & $\mathrm{T} 2$ & 0 & $\mathrm{BDL}$ & $\mathrm{BDL}$ & $\mathrm{BDL}$ & - & - & $\mathrm{BDL}$ & $\mathrm{BDL}$ & - \\
\hline & & 1 & $2.8 \pm 0.6 \mathrm{a} \mathrm{A}^{1}$ & $2.3 \pm 1.1 \mathrm{abcB}^{1}$ & $4.0 \pm 1.8 \mathrm{bA}^{1}$ & - & - & $3.0 \pm 0.4 b A^{1}$ & $72.4 \pm 20.1 \mathrm{aB}^{1}$ & 1 \\
\hline & & 3 & $2.5 \pm 0.5 \mathrm{aB}^{1}$ & $2.8 \pm 0.7 a A^{1}$ & $3.4 \pm 1.6 \mathrm{bA}^{1}$ & $1.6 \pm 0.3 b$ & - & $2.9 \pm 0.6 b c d B=1$ & $126.9 \pm 41.4 \mathrm{aB}^{1}$ & 3 \\
\hline & T3 & 0 & $\mathrm{BDL}$ & $\mathrm{BDL}$ & $\mathrm{BDL}$ & - & - & BDL & BDL & - \\
\hline
\end{tabular}

Values followed by the same letter did not significantly differ: lower case letters show indicate the difference of values in accumulation and translocation ind same plant species and growth period (LSD, $p<0.05)$; capital letters indicate the difference of values in accumulation and translocation index among plant $s$ treatment (LSD: $p<0.05$ ); and numbers indicate the difference of values in accumulation and translocation index among growth periods within the same pla 


\begin{tabular}{|c|c|c|c|c|c|c|c|c|c|c|}
\hline & & 1 & $2.7 \pm 0.9 a A^{1}$ & $2.8 \pm 0.9 a A^{1}$ & $4.2 \pm 1.4 \mathrm{bA}^{1}$ & - & - & $3.2 \pm 0.3 b A^{1}$ & $68.4 \pm 29.2 a A^{1}$ & 1 \\
\hline & & 3 & $2.4 \pm 0.5 \mathrm{aC}^{1}$ & $3.2 \pm 0.4 \mathrm{a} \mathrm{A}^{1}$ & $3.4 \pm 1.7 a b A^{2}$ & - & - & $3.0 \pm 0.7 a b B^{1}$ & $119.1 \pm 62.0 \mathrm{aA}{ }^{1}$ & 5 \\
\hline & $\mathrm{T} 4$ & 0 & BDL & $\mathrm{BDL}$ & $\mathrm{BDL}$ & - & - & $\mathrm{BDL}$ & $\mathrm{BDL}$ & - \\
\hline & & 1 & $2.5 \pm 1.1 \mathrm{abA}^{1}$ & $2.6 \pm 1.1 \mathrm{abcA}^{1}$ & $4.6 \pm 1.3 b^{1}$ & - & - & $3.2 \pm 0.3 b A^{1}$ & $46.1 \pm 16.2 \mathrm{aB}^{2}$ & 1 \\
\hline & & 3 & $1.9 \pm 0.6 b_{c d B}{ }^{2}$ & $3.0 \pm 0.6 \mathrm{aA}^{1}$ & $3.8 \pm 1.6 a b B^{1}$ & $6.0 \pm 4.9 \mathrm{ab}$ & - & $2.9 \pm 0.6 b_{c d B}{ }^{1}$ & $152.6 \pm 40.4 a A^{1}$ & 1 \\
\hline & T5 & 0 & $\mathrm{BDL}$ & $\mathrm{BDL}$ & $\mathrm{BDL}$ & - & - & $\mathrm{BDL}$ & $\mathrm{BDL}$ & - \\
\hline & & 1 & $1.9 \pm 1.2 b^{1}$ & $2.6 \pm 0.9 a b B^{1}$ & $15.0 \pm 10.2 \mathrm{a} \mathrm{A}^{1}$ & - & $5.4 \pm 3.5 a A^{1}$ & $67.1 \pm 36.8 \mathrm{a} \mathrm{A}^{2}$ & $6.4 \pm 2.5 \mathrm{aA}^{1}$ & 0 \\
\hline & & 3 & $2.3 \pm 0.6 a b B^{1}$ & $2.9 \pm 0.5 \mathrm{aA} \mathrm{A}^{1}$ & $2.7 \pm 1.6 \mathrm{cB}^{2}$ & - & - & $2.6 \pm 0.6 \mathrm{cdA} \mathrm{A}^{1}$ & $132.5 \pm 30.0 \mathrm{aAB}{ }^{1}$ & 8 \\
\hline & T6 & 0 & $\mathrm{BDL}$ & $\mathrm{BDL}$ & $\mathrm{BDL}$ & - & - & $\mathrm{BDL}$ & $\mathrm{BDL}$ & - \\
\hline & & 1 & $2.7 \pm 0.7 a A B^{1}$ & $2.3 \pm 1.2 \mathrm{bcdB}{ }^{1}$ & $4.6 \pm 1.5 \mathrm{bA}^{1}$ & - & - & $3.2 \pm 0.3 b^{1}$ & $62.2 \pm 26.4 \mathrm{a} \mathrm{A}^{1}$ & 1 \\
\hline & & 3 & $1.6 \pm 0.5 \mathrm{dC}^{2}$ & $2.8 \pm 0.6 a A^{1}$ & $3.3 \pm 1.6 \mathrm{bcB}^{2}$ & $5.1 \pm 1.1 \mathrm{bc}$ & - & $2.6 \pm 0.5 \mathrm{~dB}^{2}$ & $154.9 \pm 86.6 a A^{1}$ & 6 \\
\hline & $\mathrm{T7}$ & 0 & $\mathrm{BDL}$ & $\mathrm{BDL}$ & $\mathrm{BDL}$ & - & - & $\mathrm{BDL}$ & $\mathrm{BDL}$ & - \\
\hline & & 1 & $2.5 \pm 1.1 \mathrm{aA}^{1}$ & $2.5 \pm 1.0 a b c A^{1}$ & $4.5 \pm 1.3 b^{1}$ & - & - & $3.2 \pm 0.4 \mathrm{bB}^{1}$ & $51.9 \pm 17.0 \mathrm{aB}^{2}$ & 1 \\
\hline & & 3 & $2.3 \pm 0.5 a b B^{1}$ & $2.9 \pm 0.6 \mathrm{aA} \mathrm{A}^{1}$ & $3.3 \pm 1.5 b c B^{2}$ & $4.9 \pm 0.4 \mathrm{bc}$ & - & $2.8 \pm 0.6 b_{c d B}^{2}$ & $129.8 \pm 26.1 \mathrm{aA}^{1}$ & 1 \\
\hline & T8 & 0 & $\mathrm{BDL}$ & $\mathrm{BDL}$ & $\mathrm{BDL}$ & - & - & $\mathrm{BDL}$ & $\mathrm{BDL}$ & - \\
\hline & & 1 & $2.7 \pm 0.8 \mathrm{aA}^{1}$ & $2.4 \pm 1.0 a_{b c B}{ }^{2}$ & $6.5 \pm 3.0 \mathrm{bA}^{2}$ & - & - & $3.9 \pm 0.8 a b A B^{1}$ & $64.9 \pm 18.0 a^{2}$ & 2 \\
\hline & & 3 & $2.5 \pm 0.5 \mathrm{aB}^{1}$ & $3.3 \pm 0.7 \mathrm{aA} \mathrm{A}^{1}$ & $4.1 \pm 1.8 \mathrm{aA} \mathrm{A}^{1}$ & $7.5 \pm 0.6 a$ & - & $3.3 \pm 0.7 a A^{1}$ & $145.5 \pm 58.9 a A^{1}$ & 1 \\
\hline & T9 & 0 & $\mathrm{BDL}$ & $\mathrm{BDL}$ & $\mathrm{BDL}$ & - & - & $\mathrm{BDL}$ & $\mathrm{BDL}$ & - \\
\hline & & 1 & $2.3 \pm 1.0 a b A^{1}$ & $2.1 \pm 1.3 \mathrm{cdB}{ }^{1}$ & $4.2 \pm 1.6 \mathrm{bA}^{1}$ & - & - & $2.9 \pm 0.4 \mathrm{bB}^{1}$ & $40.0 \pm 12.8 \mathrm{aB}^{2}$ & 1 \\
\hline & & 3 & $2.1 \pm 0.4 a b c B^{1}$ & $2.7 \pm 0.4 \mathrm{aB}^{1}$ & $3.5 \pm 1.4 \mathrm{abB}^{2}$ & - & - & $2.8 \pm 0.6 b c d B=1$ & $175.1 \pm 28.5 \mathrm{a} \mathrm{A}^{1}$ & 4 \\
\hline A. & $\mathrm{T} 1$ & 0 & $\mathrm{BDL}$ & $\mathrm{BDL}$ & $\mathrm{BDL}$ & - & - & $\mathrm{BDL}$ & $\mathrm{BDL}$ & - \\
\hline & & 1 & $2.8 \pm 0.3 a b c A B^{1}$ & $2.7 \pm 0.6 a b B^{1}$ & $3.6 \pm 0.4 \mathrm{aB}^{1}$ & - & - & $3.0 \pm 0.2 a^{2} B^{2}$ & $33.4 \pm 8.0 \mathrm{bcB}^{1}$ & 3 \\
\hline & & 3 & $2.6 \pm 0.7 \mathrm{bB}^{1}$ & $3.1 \pm 0.2 \mathrm{abA}^{1}$ & $4.5 \pm 0.9 \mathrm{abA} \mathrm{A}^{1}$ & - & - & $3.4 \pm 0.2 b A^{1}$ & $81.0 \pm 38.5 a b c B^{1}$ & 3 \\
\hline & $\mathrm{T} 2$ & 0 & $\mathrm{BDL}$ & $\mathrm{BDL}$ & $\mathrm{BDL}$ & - & - & $\mathrm{BDL}$ & $\mathrm{BDL}$ & - \\
\hline & & 1 & $3.2 \pm 0.3 a b A^{1}$ & $3.0 \pm 0.3 \mathrm{a} \mathrm{A}^{1}$ & $3.3 \pm 0.1 \mathrm{abA}^{2}$ & - & - & $3.2 \pm 0.2 \mathrm{aA} \mathrm{A}^{2}$ & $35.1 \pm 9.7 b c c^{2}$ & 1 \\
\hline & & 3 & $3.9 \pm 0.5 \mathrm{abA}^{1}$ & $3.1 \pm 0.3 a b A^{1}$ & $4.0 \pm 0.2 b c A^{1}$ & - & - & $3.7 \pm 0.1 \mathrm{abA}{ }^{1}$ & $91.2 \pm 35.6 a b B^{1}$ & 1 \\
\hline & T3 & 0 & $\mathrm{BDL}$ & $\mathrm{BDL}$ & $\mathrm{BDL}$ & - & - & $\mathrm{BDL}$ & $\mathrm{BDL}$ & - \\
\hline & & 1 & $2.7 \pm 0.3 b_{c} A^{2}$ & $2.2 \pm 1.2 \mathrm{bA}^{1}$ & $3.4 \pm 0.4 a b B^{1}$ & - & - & $2.8 \pm 0.3 b B^{2}$ & $46.4 \pm 15.7 a b A^{1}$ & 2 \\
\hline & & 3 & $4.2 \pm 0.3 \mathrm{aB}^{1}$ & $2.9 \pm 0.4 a_{b A^{1}}$ & $3.5 \pm 0.5 \mathrm{cA}^{1}$ & - & - & $3.5 \pm 0.2 \mathrm{abA}^{1}$ & $35.0 \pm 20.0 \mathrm{cB}^{1}$ & 1 \\
\hline & $\mathrm{T} 4$ & 0 & $\mathrm{BDL}$ & $\mathrm{BDL}$ & $\mathrm{BDL}$ & - & - & $\mathrm{BDL}$ & $\mathrm{BDL}$ & - \\
\hline & & 1 & $2.4 \pm 0.5 c A^{1}$ & $2.6 \pm 0.3 a b A^{1}$ & $4.0 \pm 0.1 a^{2}$ & - & - & $3.0 \pm 0.2 a B^{2}$ & $43.2 \pm 16.2 \mathrm{abcB}^{1}$ & 5 \\
\hline & & 3 & $3.2 \pm 0.9 \mathrm{abA}^{1}$ & $3.0 \pm 0.2 \mathrm{abA}^{1}$ & $5.0 \pm 0.7 a A^{1}$ & - & - & $3.8 \pm 0.2 \mathrm{a} \mathrm{A}^{1}$ & $41.7 \pm 13.5 \mathrm{cB}^{1}$ & 2 \\
\hline & T5 & 0 & $\mathrm{BDL}$ & $\mathrm{BDL}$ & $\mathrm{BDL}$ & - & - & $\mathrm{BDL}$ & $\mathrm{BDL}$ & - \\
\hline & & 1 & $3.4 \pm 0.4 \mathrm{aA} \mathrm{A}^{1}$ & $3.3 \pm 0.4 a A^{1}$ & $2.7 \pm 0.7 b B^{2}$ & - & - & $3.1 \pm 0.4 a b A^{1}$ & $27.1 \pm 6.9 \mathrm{cB}^{1}$ & 2 \\
\hline & & 3 & $3.5 \pm 0.8 \mathrm{abA}{ }^{1}$ & $2.6 \pm 0.5 b A^{1}$ & $4.7 \pm 1.1 \mathrm{abA}^{1}$ & - & - & $3.6 \pm 0.5 a b A^{1}$ & $54.1 \pm 52.8 \mathrm{bcB}^{1}$ & 1 \\
\hline & T6 & 0 & $\mathrm{BDL}$ & $\mathrm{BDL}$ & $\mathrm{BDL}$ & - & - & $\mathrm{BDL}$ & $\mathrm{BDL}$ & - \\
\hline & & 1 & $2.4 \pm 0.5 \mathrm{cB}^{1}$ & $2.7 \pm 0.2 a_{b} A^{1}$ & $3.3 \pm 1.0 a B^{1}$ & - & - & $2.8 \pm 0.2 a^{2} B^{2}$ & $44.2 \pm 16.2 a b c A^{1}$ & 3 \\
\hline & & 3 & $3.0 \pm 1.4 a b B^{1}$ & $2.9 \pm 0.3 a b A^{1}$ & $4.5 \pm 0.2 \mathrm{abA}^{1}$ & - & - & $3.4 \pm 0.3 b A^{1}$ & $49.9 \pm 27.1 b^{\prime} B^{1}$ & 1 \\
\hline
\end{tabular}

Values followed by the same letter did not significantly differ; lower case letters show indicate the difference of values in accumulation and translocation ind same plant species and growth period (LSD, $p<0.05)$; capital letters indicate the difference of values in accumulation and translocation index among plant $s$ treatment (LSD: $p<0.05$ ); and numbers indicate the difference of values in accumulation and translocation index among growth periods within the same pla 


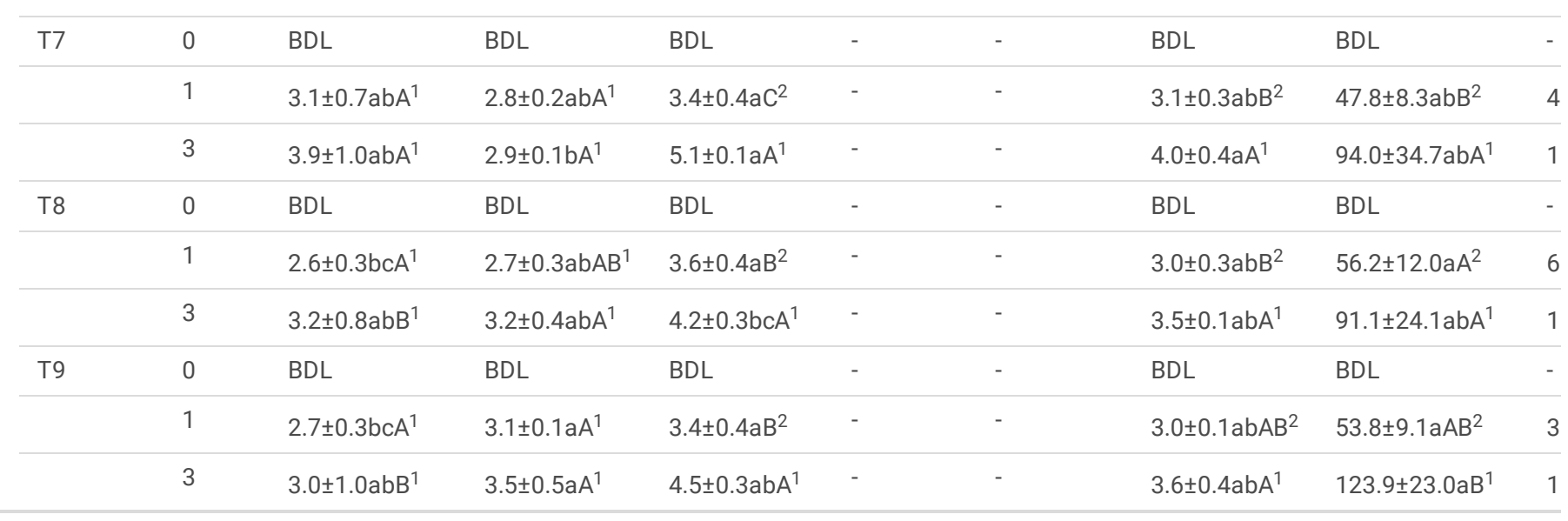

Values followed by the same letter did not significantly differ; lower case letters show indicate the difference of values in accumulation and translocation ind same plant species and growth period (LSD, $p<0.05)$; capital letters indicate the difference of values in accumulation and translocation index among plant $s$ treatment (LSD: $p<0.05)$; and numbers indicate the difference of values in accumulation and translocation index among growth periods within the same pla

After harvest, the descending order of $\mathrm{Cd}$ accumulation in the plant tissues was generally as follows: leaves $>$ roots $>$ stems for jatropha and roots $>$ stems $\approx$ leaves for cassava and acacia, respectively. Cd concentrations in roots were higher in acacia across all treatments $\left(3.5\right.$ to $\left.5.1 \mathrm{mg} \mathrm{kg}{ }^{-1}\right)$, whereas cassava and jatropha had lower accumulation with narrower ranges ( 2.7 to $4.1 \mathrm{mg} \mathrm{kg}^{-1}$ and 2.2 to $3.9 \mathrm{mg} \mathrm{kg}^{-1}$, respectively). All amended treatments showed a decrease in $\mathrm{Cd}$ accumulation by the roots of cassava to some extent, which could be attributed to the dilution effects of Cd concentration in root tissues and plant biomass. Furthermore, T5 treatment resulted in the largest decrease after three months of growth, which was approximately 5.6 times lower than the first month of growth $(p<0.05)$. Higher GRDB values in this crop plant species were most likely due to increased plant biomass, which decreased Cd concentrations in roots. All soil amendments increased Cd accumulation by shoots and roots in jatropha and acacia by 1.3 to 1.7 times and 1 to 1.7 times, respectively. Cadmium levels in soil have been demonstrated in numerous studies to rise with time, leading Cd levels in plant tissues to rise as well ${ }^{28}$. Many studies have also demonstrated that jatropha can be used as an accumulator for a variety of heavy metals; nevertheless, it is an unsuitable accumulator for all heavy metals because it accumulates some heavy metals at extremely low quantities in shoots. Furthermore, other reports claimed that this crop plant may absorb and accumulate substantial Cd concentrations in its roots $\left(3.2\right.$ to $\left.8.6 \mathrm{mg} \mathrm{kg}^{-1}\right)$. This might indicate the effects of organic amendments on heavy metal stability in contaminated soil ${ }^{29}$. In this study, shoots of jatropha showed roughly 1 to 2.6 times higher Cd concentrations than roots when Cd concentrations were compared between plant tissues from all treatments. Plants in the T2, T4, and T9 treatments, on the other hand, showed somewhat greater Cd levels in their roots than in their shoots.

The effects of different organic amendments on Cd accumulation were linked to the physicochemical properties of soils. For example, adding animal manure and biochar to soil reduced $\mathrm{Cd}$ mobility and enhanced $\mathrm{Cd}$ stabilization in soil because the amendments increased Cd sorption by increasing soil pH and $\mathrm{CEC}$, resulting in decreased Cd bioavailability and translocation in plant tissues ${ }^{30}$. In this study, leonardite has a low pH (2.6) and a high OM (20.1\%); however, it has the potential to decrease $\mathrm{Cd}$ mobility because $\mathrm{OM}$ has the potential to produce organic ligands that bind heavy metal ions to the surface of soil colloid and form stable complexes ${ }^{1}$. Compared with other treatments, SLBM in the T3 treatment for acacia exhibited remarkably low Cd concentrations in the roots and stems ( $3.5 \mathrm{mg} \mathrm{kg}^{-1}$ and $2.9 \mathrm{mg} \mathrm{kg}^{-1}$, respectively); nevertheless, the greatest Cd concentration $\left(4.2 \mathrm{mg} \mathrm{kg}^{-1}\right)$ was found in the leaves $(p<0.05)$. When the Cd concentrations in whole plant tissues of the study plants were considered, acacia and cassava showed remarkably low Cd concentrations in T3 treatment (3.5 $\mathrm{mg} \mathrm{kg}^{-1}$ and $3 \mathrm{mg} \mathrm{kg}^{-1}$, respectively), which followed a pattern similar to the Cd uptake values (35 mg plant ${ }^{-1}$ and $119.1 \mathrm{mg} \mathrm{plant}^{-1}$, respectively). The T8 treatment, on the other hand, had the lowest Cd uptake value for jatropha (127.8 $\left.\mathrm{mg} \mathrm{plant}^{-1}\right)$, as its value was based on Cd content and biomass production of the whole plant tissue. SLVC in T4 treatment for acacia substantially elevated Cd concentrations in roots $\left(5 \mathrm{mg} \mathrm{kg}^{-1} ; p<0.05\right)$; although having a high OM content (9.9\%). This might be because leonardite application reduces soil pH, as seen by an acidic soil in the $\mathrm{T} 4$ treatment (pH 5.5), resulting in increased $\mathrm{Cd}$ mobility in the soil and bioavailability. Despite the fact that BMVC had soil pH near neutral ( $\mathrm{pH} 6.8)$ and high OM content (9.6\%), the Cd concentration in the roots of acacia was greatest in the T7 treatment $\left(5.1 \mathrm{mg} \mathrm{Cd} \mathrm{kg}^{-1} ; p<0.05\right)$. To reach the considerably low Cd concentrations in plant tissues found in the T3 treatment, soil pH may need to be adjusted to a slightly alkaline level. However, high OM contents (8.6\%) in T3 treatment might be a key factor in reducing the bioavailability of the study plant. If $\mathrm{Cd}$ remediation is the main goal, BMBM in T9 treatment may be the best option, because all study plants showed substantial Cd uptake values of $187.9 \mathrm{mg} \mathrm{plant}^{-1}, 175.1 \mathrm{mg} \mathrm{plant}^{-1}$, and $123.9 \mathrm{mg} \mathrm{plant}^{-1}$ for jatropha, cassava, and acacia, respectively.

Jatropha seeds in the field had to wait for roughly 2 to $3 \mathrm{~m}$ (height) for harvesting, which is similar to the time frame in this pot study. However, jatropha seeds were only found in T2 and T4 treatments. After harvest, jatropha seeds have been discovered, which are presumed to be edible plant parts; nevertheless, seeds of jatropha are often reported to be toxic. In this pot study, the bone meal in the T2 treatment constituted a suitable soil amendment for promoting growth until seed production. The ability of cassava roots to produce and accumulate starch is dependent on the harvesting period, which is often long or approximately 9 to 12 months in agricultural areas in Thailand. Cassava tubers were found in many pot treatments such as T2, T4, T6, T7, and T8, indicating that many local soil amendments can be applied to promote the growth and production of cassava. However, they were young tubers that could not yet be consumed.

Cadmium concentrations in those edible plant parts were similarly found to be low; however, they were higher than the CODEX Alimentarius Commission, Joint FAO/WHO Food Standard Program's standard threshold $\left(>0.4 \mathrm{mg} \mathrm{kg}^{-1}\right)^{31}$.

Phytomanagement of $\mathrm{Cd}$ by the study plants. All of the plants examined under various treatments had TF values $<1$, with the values changing slightly from the first to the third month of growth $(p>0.05)$ (Table 3$)$, indicating that $\mathrm{Cd}$ was retained in plant tissues from roots to shoots. This may point to a

Page $11 / 15$ 
characteristic of the excluder phenotype that makes it suitable for phytostabilization ${ }^{14}$. From the first to the third month of growth, cassava exhibited 1.6 to 6.3 times higher BCFR values, but jatropha and acacia had lower BCFR values (Table 3). In this study, BCFR values for all study plants from all treatments were typically $>1$. Furthermore, the TF value for jatropha in the T5 treatment after harvest was $>1$, but the BCFR value was low ( 0.8$)$. According to related findings, jatropha is a perennial bioenergy crop that can be used for phytoextraction ${ }^{32}$. This perennial plant cultivated in mining soil mixed with peat moss translocated large amounts of metals from the roots to the aerial portions, which is a crucial characteristic of accumulator and hyperaccumulator crop plants $^{33}$. However, jatropha in this study cannot be considered a hyperaccumulator of Cd because their TF values are generally less than one and their BCFR values are greater than one, while their $\mathrm{Cd}$ accumulation levels did not meet Baker and Brook's criteria ${ }^{34}$, which require $\mathrm{Cd}$ levels in shoots to be greater than 100. Based on the ability for $\mathrm{Cd}$ accumulation in roots or tubers by the study plants, cassava and acacia were classified as Cd excluders, indicating that these plants can avoid importing metals to aerial parts. However, cassava peels may be the primary storage organ for heavy metals such as $\mathrm{Pb}^{35}$.

Plants suited for phytomanagement in heavy metal-contaminated areas may not have a significant capacity to accumulate high levels of heavy metals, but they should have benefits as a product rather than edible purposes. According to Robinson et al. ${ }^{36}$, phytomanagement strategy is a combination of using the benefits of phytoremediation and crop production. The potential bioenergy crops or non-edible crops, for example, can uptake and accumulate heavy metals in various plant organs; yet, plant tissues and crude extracts have diverse benefits for multi-purposes such as biofuel, fiber, paper and wood. Cassava is another appealing bioenergy crop for the reclamation of metal-polluted soils because this tuber crop is easy to cultivate in tropical and subtropical areas, and cassava tubers contain a high content of starch that could be commercially used as a renewable feedstock to produce ethanol fuel for transportation ${ }^{37}$. Because jatropha has advantageous properties like quick propagation, rapid growth, and drought tolerance, it can be widely grown in various locations globally 38 . This crop species offers a great promise for long-term industrial application as a multi-purpose plant whose components could be used as fertilizer, insecticide, soap, medicine, and energy. Despite the fact that cassava or jatropha is not a non-hyperaccumulator, they are non-food bioenergy crops that are grown in a variety of harsh environments, including heavy metal-contaminated areas ${ }^{37}$. Jatropha demonstrated a great capacity for phytoremediation of several heavy metals (iron ( $\mathrm{Fe}$ ), aluminum ( $\mathrm{Al}$ ), $\mathrm{Cr}$, manganese (Mn), and $\mathrm{Cu}$ ) from fly ash in greenhouse studies, as it could increase heavy metal uptake by $117 \%$ in the roots, $62 \%$ in the stems, and $86 \%$ in the leaves, respectively ${ }^{37,39}$. Jatropha and cassava, which may be grown in heavily Cd-contaminated soils and used for renewable energy or biodiesel, are considered suitable for long-term use and rehabilitation of heavy metal-contaminated lands ${ }^{37,40}$.

The plant growth data of acacia compared with that of other study plants, revealed that this dicotyledon perennial plant had the highest dry biomass and GRDB, as well as low $\mathrm{Cd}$ accumulation and no toxicity effects. Because acacia, an $\mathrm{N}$-fixing plant, can fix atmospheric $\mathrm{N}_{2}$ in symbiotic root nodules and it does

not have any major pest concerns, and increased plant growth can be expected ${ }^{41}$. After harvest, acacia wood is very heavy, hard, robust, and durable, meaning it can be used for furniture, doors, and window frames ${ }^{42}$. Perennial bioenergy crops require fewer nutrient inputs and have higher lignin and cellulose content than annual crop biomass ${ }^{43}$. Therefore, it could be considered that acacia can be cultivated over a large area such as in a contaminated area and that it has substantial biomass, making it more suitable for use as an energy plant in Cd-contaminated lands.

\section{Conclusions}

Plant biomass found in contaminated areas accumulates high quantities of heavy metals, rendering it inappropriate for human consumption or feeding to animals; however, plant biomass can be anaerobically digested or burned to be employed as energy production. Bioenergy crops can eventually replace edible plants in the field, particularly rice, while also allowing for the long-term use and rehabilitation of heavy metal-contaminated soils, resulting in substantial incomes to local communities and farmers. Furthermore, perennial plants, e.g., acacia and jatropha have low cultivation requirements and are a cost-effective long-term option, making it a viable option for phytomanagement in mining areas. However, soil amendments, e.g., bone meal, leonardite, and bat manure are required to improve plant growth and development, as well as $\mathrm{Cd}$ mobility in soil, resulting in a lower bioavailability of Cd for bioenergy crops.

\section{Declarations}

\section{Acknowledgment}

This research received funding support from the NSRF via the Program Management Unit for Human Resources \& Institutional Development, Research, and Innovation (grant number B05F630047).

\section{Author contributions}

P.T., K.H., and W.M. conceived the idea and designed the project. P.T. and K.H. conducted the study, analyzed the data, interpreted the results, and wrote the manuscript. W.M. gave supervision to this study, edited and approved the overall final version of the manuscript. All authors discussed the data and commented on the manuscript.

\section{Completing interests}

The authors declare no competing interests.

\section{References}

1. Saengwilai, P. \& Meeinkuirt, W. Cadmium (Cd) and zinc (Zn) accumulation by Thai rice varieties and health risk assessment in a Cd-Zn co-contaminated paddy field: Effect of soil amendments. Environ. Geochem. Health. <background-color:\#FFCC66;bvertical-align:super; >43</background-

color:\#FFCC66;bvertical-align:super;>, 3659-3674 (2021). 
2. Lee, K. K., Cho, H. S., Moon, Y. C., Ban, S. J. \& Kim, J. Y. Cadmium and lead uptake capacity of energy crops and distribution of metals within the plant structures. KSCE J. Civ. Eng. <background-color:\#FFCC66;bvertical-align:super;>17</background-color:\#FFCC66;bvertical-align:super;>(1), 44-50 (2013).

3. Sharma, S., Tiwari, S., Hasan, A., Saxena, V. \& Pandey, L. M. Recent advances in conventional and contemporary methods for remediation of heavy metalcontaminated soils. 3 Biotech<background-color:\#FFCC66;bvertical-align:super;>8</background-color:\#FFCC66;bvertical-align:super;>(4), 216. <background-color:\#CFBFB1;uvertical-align:super;>https://doi:10.1007/s13205-018-1237-8</background-color:\#CFBFB1;uvertical-align:super;><uverticalalign:super;></uvertical-align:super; $>(2018)$.

4. Nedjimi, B. Phytoremediation: a sustainable environmental technology for heavy metals decontamination. SN Appl. Sci. <backgroundcolor:\#FFCC66;bvertical-align:super;>3</background-color:\#FFCC66;bvertical-align:super;>, 286. https://doi.org/10.1007/s42452-021-04301-4 (2018).

5. Saengwilai, P., Meeinkuirt, W., Pichtel, J. \& Koedrith P. Influence of amendments on Cd and Zn uptake and accumulation in rice (Oryza sativa L.) in contaminated soil. Environ. Sci. Pollut. Res. <background-color:\#FFCC66;bvertical-align:super;>24</background-color:\#FFCC66;bvertical-align:super;>(18), 15756-15767 (2017).

6. Saengwilai, P., Meeinkuirt, W., Phusantisampan, T. \& Pichtel, J. Immobilization of cadmium in contaminated soil using organic amendments and its effects on rice growth performance. Exp. Health<background-color:\#FFCC66;bvertical-align:super;>12</background-color:\#FFCC66;bvertical-align:super;>, 295-306 (2020).

7. Zgorelec, Z., Bilandzija, N., Knez, K., Galic, M. \& Zuzul, S. Cadmium and mercury phytostabilization from soil using Miscanthus $\times$ giganteus. Sci. Rep. <background-color:\#FFCC66;bvertical-align:super;>10</background-color:\#FFCC66;bvertical-align:super;>, 6685. https://doi.org/10.1038/s41598-02063488-5 (2020).

8. Chen, Y., Li, X. \& Shen, Z. Leaching and uptake of heavy metals by ten different species of plants during an EDTA-assisted phytoextraction process. Chemosphere<background-color:\#FFCC66;bvertical-align:super;>57</background-color:\#FFCC66;bvertical-align:super;>, 187-196 (2004).

9. Jeanne, B. A. et al. Enhancement of phytoremediation efficiency of Acacia mangium using earthworms in metal-contaminated soil in Bonoua, Ivory Coast. Afr. J. Biotechnol.<bivertical-align:baseline;></bivertical-align:baseline;><background-color:\#FFCC66;bvertical-align:super;>18</backgroundcolor:\#FFCC66;bvertical-align:super;>(27), 622-631 (2019).

10. Alcantara, H. J. P. et al. Localization of mercury and gold in cassava (Manihot esculenta Crantz). Environ. Sci. Pollut. Res. <backgroundcolor:\#FFCC66;bvertical-align:super;>27</background-color:\#FFCC66;bvertical-align:super;>, 18498-18509 (2020).

11. APHA AWWA and WEF (American Public Health Association, American Water Works Association and Water Environment Federation). Standard Methods for the Examination of Water and Wastewater (American Public Health Association, 2005)

12. Blaylock, M. J. et al. Enhanced accumulation of $\mathrm{Pb}$ in Indian mustard by soil applied chelating agents. Environ. Sci. Technol.<bivertical-align:baseline;> </bivertical-align:baseline;><background-color:\#FFCC66;bvertical-align:super;>31</background-color:\#FFCC66;bvertical-align:super;>, 860-865 (1997).

13. Zou, T., Li, T., Zhang, X., Yu, H. \& Huang, H. Lead accumulation and phytostabilization potential of dominant plant species growing in a lead-zinc tailing. Environ. Earth Sci. <background-color:\#FFCC66;bvertical-align:super;>65</background-color:\#FFCC66;bvertical-align:super;>, 621-630 (2012).

14. Phusantisampan, T., Meeinkuirt, W., Saengwilai, P., Pichtel, J. \& Chaiyarat, R. Phytostabilization potential of two ecotypes of Vetiveria zizanioides in cadmium-contaminated soils: greenhouse and field experiments. Environ. Sci. Pollut. Res.<bivertical-align:baseline;></bivertical-align:baseline;> <background-color:\#FFCC66;bvertical-align:super;>23</background-color:\#FFCC66;bvertical-align:super;>, 20027-20038 (2016).

15. Thongchai, A., Meeinkuirt, W., Taeprayoon, P. \& Pichtel, J. Soil amendments for cadmium phytostabilization by five marigold cultivars. Environ. Sci. Pollut. Res. <background-color:\#FFCC66;bvertical-align:super;>26</background-color:\#FFCC66;bvertical-align:super;>, 8737-8747 (2019).

16. Chakraborty, K. \& Mistri, B. Importance of soil texture in sustenance of agriculture: A study in Burdwan-I C. D. Block, Burdwan, West Bengal. East. Geogr. <background-color:\#FFCC66;bvertical-align:super;>1</background-color:\#FFCC66;bvertical-align:super;>, 475-482 (2015).

17. Moreno, J. L., Ondoño, S., Torres, I. \& Bastida, F. Compost, leonardite, and zeolite impacts on soil microbial community under barley crops. J. Soil Sci. Plant Nutr. <background-color:\#FFCC66;bvertical-align:super;>17</background-color:\#FFCC66;bvertical-align:super;>, 214-230 (2017).

18. Andriolo, J. L. et al. Growth and yield of lettuce plants under salinity. Hortic. Bras. <background-color:\#FFCC66;bvertical-align:super;>23</backgroundcolor:\#FFCC66;bvertical-align:super;>(4), 931-934 (2005).

19. Khan, N. et al. Critical values of alternative organic amendments on kiwi seedling growth. Soil Sci. Plant Nutr. <background-color:\#FFCC66;bverticalalign:super;>64</background-color:\#FFCC66;bvertical-align:super;>(6), 774-781 (2018).

20. Guo, Y. \& Rockstraw, A. D. Physicochemical properties of carbons prepared from pecan shell by phosphoric acid activation. Bioresour. Technol. <background-color:\#FFCC66;bvertical-align:super;>98</background-color:\#FFCC66;bvertical-align:super;>, 1513-1521 (2007).

21. Simmons, R. W., Pongsakul, P., Saiyasitpanich, D. \& Klinphoklap, S. Elevated levels of cadmium and zinc in paddy soils and elevated levels of cadmium in rice grain downstream of a zinc mineralized area in Thailand: Implications for public health. Environ. Geochem. Health <backgroundcolor:\#FFCC66;bvertical-align:super;>27</background-color:\#FFCC66;bvertical-align:super;>, 501-511 (2005).

22. Nelson, N. O. \& Janke, R. R. Phosphorus sources and management in organic production systems. Horttechnology <background-color:\#FFCC66;bverticalalign:super;>17</background-color:\#FFCC66;bvertical-align:super;>(4), 442-454 (2007).

23. Sinha, A., et al. Optimizing organic and mineral amendments to Jatropha seed cake to increase its agronomic utility as organic fertilizer. Arch. Agro. Soil Sci. <background-color:\#FFCC66;bvertical-align:super;>57</background-color:\#FFCC66;bvertical-align:super;>(2), 193-222 (2011).

24. Butnan, S., Toomsan, B. \& Vityakon, P. Organic and chemical fertilizers have varied effects on tomato growth in a sandy soil. Khon Kaen Agr. J. <background-color:\#FFCC66;bvertical-align:super;>47</background-color:\#FFCC66;bvertical-align:super;>(Suppl. 1), 1705-1710 (2019)

25. Isuwan, A. Agronomic traits and fruit quality of pineapple with different levels of chicken manure application. Silpakorn U.<backgroundcolor:\#CCFF99;idirection:rtl;vertical-align:super;></background-color:\#CCFF99;idirection:rtl;vertical-align:super;> Sci. Technol. <background-

Page 13/15 
color:\#FFCC66;bvertical-align:super;>8</background-color:\#FFCC66;bvertical-align:super;>(1), 67-73 (2014).

26. Suwor, P., Jeakkhajorn, S. \& Kramchote, S. Effects of different compost manures application on growth of lettuces (Lactuca sativa L.). Int. J. Agri. Technol. <background-color:\#FFCC66;bvertical-align:super;>16</background-color:\#FFCC66;bvertical-align:super;>(5), 1257-1266 (2020).

27. Meeinkuirt, W., Kruatrachue, M., Tanhan, P., Chaiyarat, R. \& Poketthitiyook, P. Phytostabilization potential of Pb mine tailings by two grass species, Thysanolaena maxima and Vetiveria zizanioides. Water Air Soil Pollut. <background-color:\#FFCC66;bvertical-align:super;>224</backgroundcolor:\#FFCC66;bvertical-align:super;>, 1750. https://doi:10.1007/s11270-013-1750-7 (2013).

28. De Maria, S., Puschenreiter, M. \& Rivelli, A. R. Cadmium accumulation and physiological response of sunflower plants to Cd during the vegetative growing cycle. Plant Soil Environ. <background-color:\#FFCC66;bvertical-align:super;>59</background-color:\#FFCC66;bvertical-align:super;>(6), 254-261 (2013).

29. Ahmadpour, P. et al. Uptake of heavy metals by Jatropha curcas L. planted in soils containing sewage sludge. American J. Appl. Sci. <backgroundcolor:\#FFCC66;bvertical-align:super;>7</background-color:\#FFCC66;bvertical-align:super;>(10), 1291-1299 (2010).

30. Hamid, Y. et al. Immobilization of cadmium and lead in contaminated paddy field using inorganic and organic additives. Sci. Rep. <backgroundcolor:\#FFCC66;bvertical-align:super;>8</background-color:\#FFCC66;bvertical-align:super;>, 17839. <background-color:\#CFBFB1;uverticalalign:super;>https://doi:10.1038/s41598-018-35881-8</background-color:\#CFBFB1;uvertical-align:super;><uvertical-align:super;></uvertical-align:super;> (2018).

31. Chunhabundit, R. Cadmium exposure and potential health risk from foods in contaminated areas, Thailand. Toxicol. Res. <backgroundcolor:\#FFCC66;bvertical-align:super;>32</background-color:\#FFCC66;bvertical-align:super;>(1), 65-72 (2016).

32. Tang, Y.-T. et al. Lead, zinc, cadmium hyperaccumulation and growth stimulation in Arabis paniculata Franch. Environ. Exp. Bot. <backgroundcolor:\#FFCC66;bvertical-align:super;>66</background-color:\#FFCC66;bvertical-align:super;>, 126-134 (2009).

33. Martin, J. F. G. et al. Metal accumulation by Jatropha curcas L. adult plants grown on heavy metal-contaminated soil. Plants $<$ backgroundcolor:\#FFCC66;bvertical-align:super;>9</background-color:\#FFCC66;bvertical-align:super;>, 418. https://doi:10.3390/plants9040418 (2020).

34. Baker, A. J. M. \& Brooks, R. R. Terrestrial higher plants which hyperaccumulate metallic elements-A review of their distribution, ecology and phytochemistry. Biorecovery <background-color:\#FFCC66;bvertical-align:super;>1</background-color:\#FFCC66;bvertical-align:super;>, 81-126 (1989).

35. Udiba, U., Udofia, U., Akpan, R. \& Antai, E. Assessment of lead (Pb) uptake and hazard potentials of cassava plant (Manihot esculentus cranz), Dareta Village, Zamfara, Nigeria. Int. Res. J. Pub. Environ. Health<background-color:\#FFCC66;bvertical-align:super;>6</background-color:\#FFCC66;bverticalalign:super;>(6), 115-126 (2019).

36. Robinson, B. H., Green, S. R., Chancerel, B., Mills, T. M. \& Clothier, B. E. Poplar for phytomanagement of boron contaminated sites. Environ. Pollut. <background-color:\#FFCC66;bvertical-align:super;>150</background-color:\#FFCC66;bvertical-align:super;>, 225-233.

37. Shen, S., Chen, J., Chang, J. \& Xia, B. Using bioenergy crop cassava (Manihot esculenta) for reclamation of heavily metal-contaminated land. Int. J. Phytoremediat. <background-color:\#FFCC66;bvertical-align:super;>22</background-color:\#FFCC66;bvertical-align:super;>(12), 1313-1320 (2020).

38. Mingyuan, L., Samsuri, A.W., Shukor, M. Y. \& Phang, L.Y. Growth Performance of Jatropha curcas cultivated on local abandoned bauxite mine soil. Sustainability <background-color:\#FFCC66;bvertical-align:super;>12</background-color:\#FFCC66;bvertical-align:super;>(19), 8263. https://doi.org/10.3390/su12198263 (2020).

39. Jamil S., Abhilash, P. C., Singh, N. \& Sharma, P. N. Jatropha curcas: A potential crop for phytoremediation of coal fly ash. J. Hazard. Mater. <backgroundcolor:\#FFCC66;bvertical-align:super;>172</background-color:\#FFCC66;bvertical-align:super;>(1), 269-275 (2009).

40. Prasad, M. N. V., Nakbanpote, W., Sebastian, A., Natthawoot, P. \& Phadermrod, C. Phytomanagement of Padaeng zinc mine waste, Mae Sot district, Tak province, Thailand in Soil remediation and plants (eds. Hakeem, K. R., Sabir, M., Ozturk, M. \& Murmut, A.) 661-687 (Academic Press, 2015).

41. Lee, S. S. Diseases and potential threats to Acacia mangium plantations in Malaysia. Unasy/va <background-color:\#FFCC66;bverticalalign:super;>217</background-color:\#FFCC66;bvertical-align:super;>(55), 3135 (2004).

42. Sharma, S. K., Kumar, P., Rao, R. V., Sujatha, M. \& Shukla S. R. Rational utilization of plantation grown Acacia mangium Willd. J. Indian Acad. Wood Sci. <background-color:\#FFCC66;bvertical-align:super;>8</background-color:\#FFCC66;bvertical-align:super;>(2), 97-99 (2011).

43. Pogrzeba, M., Krzyzak, J., Rusinowski, S., Mccalmont, J. P. \& Jensen, E. Energy crop at heavy metal-contaminated arable land as an alternative for food and feed production: Biomass quantity and quality in Plant metallomics and functional omics (ed. Sablok, G.) 1-21 (Springer, 2019).

\section{Figures}




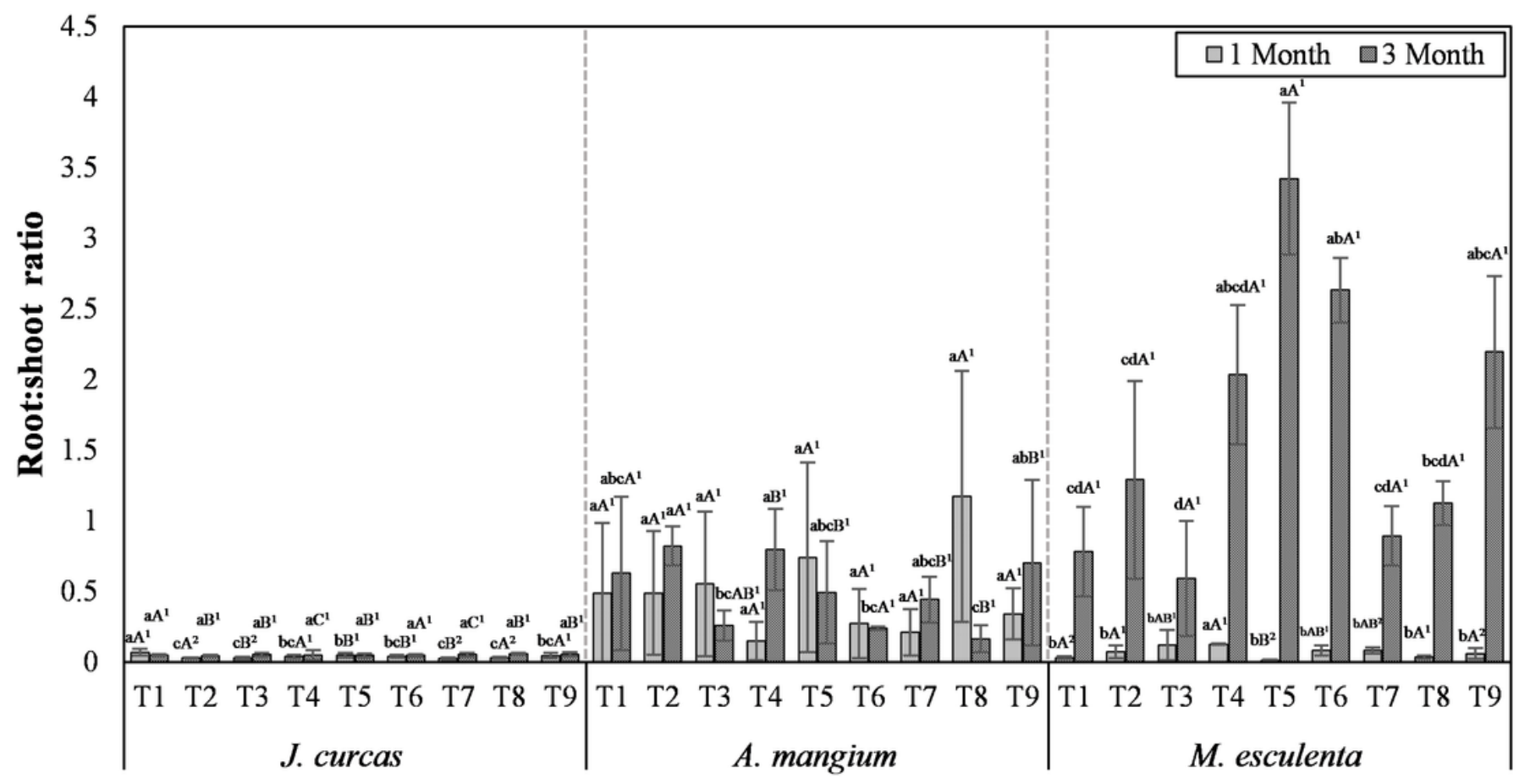

Figure 1

Root/shoot ratio of $\mathrm{Cd}$ in the study plants grown in a pot. Values followed by the same letter are not different; small letters show the differences in R/S ratio values among treatments within the same plant species and growth period (LSD: $p<0.05$ ); capital letters show the differences in R/S ratio values among plant species within the same treatment (LSD: $p<0.05)$; and numbers indicate the differences in R/S ratio values between growth periods within the same plant and treatment. 